\title{
Interpreting a $750 \mathrm{GeV}$ diphoton resonance
}

\author{
Rick S. Gupta, ${ }^{a}$ Sebastian Jäger ${ }^{a, b}$ Yevgeny Kats, ${ }^{a}$ Gilad Perez ${ }^{a}$ and \\ Emmanuel Stamou ${ }^{a}$ \\ ${ }^{a}$ Department of Particle Physics and Astrophysics, Weizmann Institute of Science, \\ Rehovot 7610001, Israel \\ ${ }^{b}$ Department of Physics and Astronomy, University of Sussex, \\ Brighton, BN1 9QH, U.K. \\ E-mail: rsgupta@weizmann.ac.il, S.Jaeger@sussex.ac.uk, \\ yevgeny.kats@weizmann.ac.il, gilad.perez@weizmann.ac.il, \\ emmanuel.stamou@weizmann.ac.il
}

ABSTRaCt: We discuss the implications of the significant excesses in the diphoton final state observed by the LHC experiments ATLAS and CMS around a diphoton invariant mass of $750 \mathrm{GeV}$. The interpretation of the excess as a spin-zero $s$-channel resonance implies model-independent lower bounds on both its branching ratio and its coupling to photons, which stringently constrain dynamical models. We consider both the case where the excess is described by a narrow and a broad resonance. We also obtain model-independent constraints on the allowed couplings and branching fractions to final states other than diphotons, by including the interplay with $8 \mathrm{TeV}$ searches. These results can guide attempts to construct viable dynamical models of the resonance. Turning to specific models, our findings suggest that the anomaly cannot be accounted for by the presence of only an additional singlet or doublet spin-zero field and the Standard Model degrees of freedom; this includes all two-Higgs-doublet models. Likewise, heavy scalars in the MSSM cannot explain the excess if stability of the electroweak vacuum is required, at least in a leading-order analysis. If we assume that the resonance is broad we find that it is challenging to find a weakly coupled explanation. However, we provide an existence proof in the form of a model with vectorlike quarks with large electric charge that is perturbative up to the $100 \mathrm{TeV}$ scale. For the narrow-resonance case a similar model can be perturbative up to high scales also with smaller charges. We also find that, in their simplest form, dilaton models cannot explain the size of the excess. Some implications for flavor physics are briefly discussed.

Keywords: Beyond Standard Model, Higgs Physics, Supersymmetric Standard Model

ARXiv EPrint: 1512.05332 


\section{Contents}

1 Introduction 1

2 Model-independent constraints 2

2.1 Implications of the excess alone 2

$\begin{array}{lll}2.2 & \text { Interplay with previous LHC searches } & 6\end{array}$

3 Models 12

$\begin{array}{lll}3.1 & \text { SM-singlet scalar } & 12\end{array}$

$\begin{array}{lll}3.1 .1 & \text { Renormalizable model } & 12\end{array}$

$\begin{array}{ll}\text { 3.1.2 Boosting } c_{\gamma}, c_{g} \text { with new vectorlike fermions } & 13\end{array}$

$\begin{array}{ll}3.1 .3 \text { A pseudoscalar } & 16\end{array}$

$\begin{array}{lll}3.1 .4 & \text { The dilaton } & 17\end{array}$

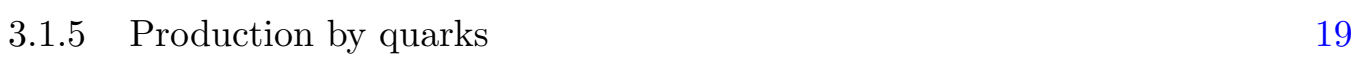

$\begin{array}{ll}3.2 & \text { Excluding the general pure 2HDM }\end{array}$

3.3 The fate of the MSSM 24

$\begin{array}{lll}\text { 3.3.1 Constraints from vacuum stability } & 26\end{array}$

$\begin{array}{lll}\text { 3.3.2 Conservative bounds on sfermion contributions } & 27\end{array}$

$\begin{array}{lll}\text { 3.3.3 Contributions from other particles and verdict } & 29\end{array}$

$\begin{array}{lll}3.3 .4 & \text { Production from quarks? } & 29\end{array}$

$\begin{array}{lll}3.3 .5 & \text { Cautionary note } & 31\end{array}$

4 Summary and outlook $\quad 31$

\section{Introduction}

Very recently, both the ATLAS and the CMS collaborations at CERN have reported mutually consistent "bumps" in the diphoton invariant mass distribution around $750 \mathrm{GeV}[1,2]$. Based on 3.2 and $2.6 \mathrm{fb}^{-1}$ of the $13 \mathrm{TeV}$ LHC data, the corresponding deviations from the background-only hypothesis have a local significance of $3.9 \sigma$ and $2.6 \sigma$ in ATLAS and CMS, respectively. The bumps are best described by a relative width $\Gamma / M \approx 6 \%$ in ATLAS [1] but a sub-resolution width in CMS [2]. However, this discrepancy is not statistically significant and we will generally present results as a function of the unknown width. The resonant excesses are suggestive of the decay of a new particle beyond the Standard Model (BSM). The kinematic properties of the events in the excess region are reported not to show significant deviations compared with events in sidebands. This disfavors significant contributions to the production from decays of yet heavier particles or associated production and motivates focusing on the case of a single production of a $750 \mathrm{GeV}$ resonance. 
The purpose of the present paper is to characterise this theoretically unexpected result and discuss its implications for some leading paradigms for physics beyond the Standard Model. It is divided into two main parts, the first of which comprises a model-independent framework that aims to equip the reader with handy formulas for interpreting both the signal and the most important resonance search constraints from existing LHC searches in the context of BSM models. We derive a number of bounds, including model-independent lower bounds on the branching ratio and partial width into photons of the hypothetical new particle. The second part investigates concrete scenarios, including the possibility of interpreting the resonance as the dilaton in a theory with spontaneous breaking of scale invariance or as a heavy Higgs scalar a two-Higgs-doublet model (2HDM). We find the properties of the observed excess to be quite constraining. In particular, a leading-order analysis suggests that the interpretation as an $s$-channel resonance, if confirmed, cannot be accommodated within the Minimal Supersymmetric Standard Model (MSSM) even under the most conservative assumptions about the MSSM parameters and the true width of the resonance; this conclusion holds if we require the absence of charge- and colour-breaking minima.

\section{Model-independent constraints}

We start by discussing what can be inferred about the new hypothetical particle from data alone. We will first describe the implications of the observed properties of the diphoton bumps, and then examine the constraints from the absence of significant excesses in resonance searches in other final states that could be sensitive to other decay modes of the same particle.

\subsection{Implications of the excess alone}

Both ATLAS and CMS observe excesses in a diphoton invariant mass region near $750 \mathrm{GeV}[1,2]$. For the purposes of this work, we will generally assume the signal contribution to be $N=20$ events for $\mathcal{L}=5.8 \mathrm{fb}^{-1}$ integrated luminosity (adding up ATLAS and CMS), but will make clear the scaling of our findings with $N$ wherever feasible. We will assume a signal efficiency (including acceptance) of $\epsilon=50 \%$, even though, in general, this does have some dependence on both the experiment and the details of the signal model.

The most straightforward signal interpretation is resonant $s$-channel production of a new unstable particle. The observed signal strength corresponds to a $13 \mathrm{TeV}$ inclusive cross section to diphotons of

$$
\sigma_{13} \times \mathrm{BR}_{\gamma \gamma} \approx 6.9 \mathrm{fb} \times\left(\frac{N}{20}\right)\left(\frac{50 \%}{\epsilon}\right)\left(\frac{5.8 \mathrm{fb}^{-1}}{\mathcal{L}_{13}}\right) .
$$

The diphoton final state precludes a spin-one interpretation due to the LandauYang theorem $[3,4]$, and we will henceforth assume spin zero. We take the mass to be $M=750 \mathrm{GeV}$; small variations have no significant impact on our findings. The shape of the excess in ATLAS may indicate a width of about $\Gamma=45 \mathrm{GeV}$ [1]. However, we will also contemplate the case of smaller width below, and discuss how our main findings depend on this. 
A minimal dynamical input is necessary to interpret the result and incorporate $8 \mathrm{TeV}$ LHC constraints. The width-to-mass ratio is small enough to justify a narrow-width approximation to the level of accuracy we aim at here. In the narrow-width limit, resonant scattering amplitudes factorize into production and decay vertices, which we parameterize by terms in a "Lagrangian" for the resonance $\mathcal{S}$,

$$
\begin{aligned}
\mathcal{L}= & -\frac{1}{16 \pi^{2}} \frac{1}{4} \frac{c_{\gamma}}{M} \mathcal{S} F^{\mu \nu} F_{\mu \nu}-\frac{1}{16 \pi^{2}} \frac{1}{4} \frac{c_{g}}{M} \mathcal{S} G^{\mu \nu, a} G_{\mu \nu}^{a} \\
& -\frac{1}{16 \pi^{2}} \frac{1}{2} \frac{c_{W}}{M} \mathcal{S} W^{-\mu \nu} W_{\mu \nu}^{+}-\frac{1}{16 \pi^{2}} \frac{1}{4} \frac{c_{Z}}{M} \mathcal{S} Z^{\mu \nu} Z_{\mu \nu}-\frac{1}{16 \pi^{2}} \frac{1}{4} \frac{c_{Z \gamma}}{M} \mathcal{S} Z^{\mu \nu} F_{\mu \nu} \\
& -\widehat{c_{W}} m_{W} \mathcal{S} W^{+\mu} W_{\mu}^{-}-\frac{1}{2} \widehat{c_{Z}} m_{Z} \mathcal{S} Z^{\mu} Z_{\mu}-\sum_{f} c_{f} \mathcal{S} \bar{f} f .
\end{aligned}
$$

In this parametrization, $M$ is the mass of the resonance $\mathcal{S}$. We emphasize that each term denotes a particular production and/or decay vertex and that the parameterization $\mathcal{L}$ does not make any assumptions about hierarchies of scales. ${ }^{1}$ If $\mathcal{S}$ is a pseudoscalar, $\widehat{c_{W}}=\widehat{c_{Z}}=0$, while all the other couplings lead to the same results as we present in this section for the scalar upon the replacements $\mathcal{S} \bar{f} f \rightarrow i \mathcal{S} \bar{f} \gamma^{5} f, X^{\mu \nu} X_{\mu \nu}^{\prime} \rightarrow X^{\mu \nu} \tilde{X}_{\mu \nu}^{\prime}$, where $X^{(\prime) \mu \nu}=F^{\mu \nu}$, $G^{\mu \nu, a}, W^{ \pm \mu \nu}, Z^{\mu \nu}$ (up to minor differences in the phase-space factors from table 1 below).

The total decay width of $\mathcal{S}$ imposes one constraint on the couplings,

$$
\frac{\Gamma}{M}=\sum_{i} \frac{\Gamma_{i}}{M}=\sum_{i} n_{i}\left|c_{i}\right|^{2} \approx 0.06
$$

where the (dimensionless) coefficients $n_{i}$ are listed in table 1 for the modes considered in the present analysis. In particular, eq. (2.3) directly implies upper bounds on the magnitude of each $c_{i}$, since observations imply that the width cannot exceed the ATLAS-preferred value of $45 \mathrm{GeV}$ by more than a factor of about two.

It is possible and convenient to represent the observed signal in terms of the branching ratios to the production mode and to $\gamma \gamma$. If a single production mode, $p$, dominates, the number of signal events, $N$, in the $13 \mathrm{TeV}$ analyses fixes the product

$$
\mathrm{BR}_{\gamma \gamma} \times \mathrm{BR}_{p}=n_{p} \frac{M}{\Gamma} \frac{N}{\epsilon x_{\mathcal{S}}^{13, p} \mathcal{L}_{13}}=\kappa_{p} \times\left(\frac{N}{20}\right)\left(\frac{45 \mathrm{GeV}}{\Gamma}\right)\left(\frac{5.8 \mathrm{fb}^{-1}}{\mathcal{L}_{13}}\right)
$$

where, for the production modes mediated by the various couplings from eq. (2.2),

$$
\begin{array}{cccccccccccc}
g g & u \bar{u} & d \bar{d} & s \bar{s} & c \bar{c} & b \bar{b} & W W & \widehat{W W} & Z Z & \widehat{Z Z} & Z \gamma & \gamma \gamma \\
\kappa_{p} \approx\{2.5,5.5,8.9, & 96,140,310, & 1600, & 16000, & 2400, & 21000, & 1400, & 170\} \times 10^{-5} .
\end{array}
$$

We used the leading-order $\sqrt{s}=13 \mathrm{TeV}$ production cross sections for $M=750 \mathrm{GeV}$,

$$
\sigma_{13}=\left|c_{p}\right|^{2} x_{\mathcal{S}}^{13, p},
$$

\footnotetext{
${ }^{1}$ In particular, the "couplings" $c_{i}$ are on-shell form factors that generally include contributions from light particles and CP-even phases due to unitarity cuts. Contributions from particles with mass $\gg M$ can be matched to a local effective Lagrangian similar to eq. (2.2). We discuss examples in section 3.
} 


\begin{tabular}{|ccc|}
\hline mode & Width coefficient $n_{i}$ & $n_{i}(\#)$ \\
\hline$\gamma \gamma$ & $\frac{1}{16(4 \pi)^{5}}$ & $1.99 \times 10^{-7}$ \\
$g g$ & $\frac{1}{2(4 \pi)^{5}}$ & $1.60 \times 10^{-6}$ \\
$q_{i} \bar{q}_{i}$ & $\frac{3}{8 \pi}$ & 0.119 \\
$\widehat{W W}$ & $\frac{1}{64 \pi} \sqrt{1-4 m_{W}^{2} / M^{2}} \frac{M^{2}}{m_{W}^{2}}\left(1-4 \frac{m_{W}^{2}}{M^{2}}+12 \frac{m_{W}^{4}}{M^{4}}\right)$ & 0.404 \\
$\widehat{Z Z}$ & $\frac{1}{128 \pi} \sqrt{1-4 m_{Z}^{2} / M^{2}} \frac{M^{2}}{m_{Z}^{2}}\left(1-4 \frac{m_{Z}^{2}}{M^{2}}+12 \frac{m_{Z}^{4}}{M^{4}}\right)$ & 0.154 \\
$W W$ & $\frac{1}{8(4 \pi)^{5}} \sqrt{1-4 m_{W}^{2} / M^{2}}\left(1-4 \frac{m_{W}^{2}}{M^{2}}+6 \frac{m_{W}^{4}}{M^{4}}\right)$ & $3.72 \times 10^{-7}$ \\
$Z Z$ & $\frac{1}{16(4 \pi)^{5}} \sqrt{1-4 m_{Z}^{2} / M^{2}}\left(1-4 \frac{m_{Z}^{2}}{M^{2}}+6 \frac{m_{Z}^{4}}{M^{4}}\right)$ & $1.82 \times 10^{-7}$ \\
$Z \gamma$ & $\frac{1}{32(4 \pi)^{5}}\left(1-\frac{m_{Z}^{2}}{M^{2}}\right)^{3}$ & $9.54 \times 10^{-8}$ \\
\hline
\end{tabular}

Table 1. Width coefficients.

where $x_{\mathcal{S}}^{13, p}$ are listed in table $2 .^{2}$ A direct consequence of eq. (2.4) is a lower bound on the branching ratio into photons,

$$
\mathrm{BR}_{\gamma \gamma}>\kappa_{p}\left(\frac{N}{20}\right)\left(\frac{45 \mathrm{GeV}}{\Gamma}\right)\left(\frac{5.8 \mathrm{fb}^{-1}}{\mathcal{L}_{13}}\right) .
$$

Note that this bound becomes more stringent if the width of the resonance is reduced.

Alternatively, the excess events fix the product of couplings

$$
\left|c_{\gamma} c_{p}\right|=\sqrt{n_{\gamma}^{-1} \frac{\Gamma}{M} \frac{N}{\epsilon x_{\mathcal{S}}^{13, p} \mathcal{L}_{13}}}=\rho_{p} \times \sqrt{\left(\frac{N}{20}\right)\left(\frac{\Gamma}{45 \mathrm{GeV}}\right)\left(\frac{5.8 \mathrm{fb}^{-1}}{\mathcal{L}_{13}}\right)},
$$

where

$$
\begin{array}{cccccccccccc}
g g & u \bar{u} & d \bar{d} & s \bar{s} & c \bar{c} & b \bar{b} & W W & \widehat{W W} & Z Z & \widehat{Z Z} & Z \gamma & \gamma \gamma \\
\rho_{p} \approx\{530, & 2.9, & 3.7 & 12, & 15 & 22, & 28000 & 84 & 49000, & 160, & 52000, & 12000\}
\end{array} .
$$

Importantly, increasing the production couplings, $c_{p}$, increases also the decay rates to the production modes. Since these compete with the $\gamma \gamma$ decay, $c_{\gamma}$ cannot be arbitrarily small. The smallest possible $\left|c_{\gamma}\right|$ corresponds to the situation where the total width is dominated by the production mode (which in particular implies $\Gamma_{\gamma \gamma} \ll \Gamma_{p}$, for production modes other than $\gamma \gamma$ ). Since the dependence on $\left|c_{p}\right|^{2}$ cancels between the production cross

\footnotetext{
${ }^{2}$ Results for VBF production, here and below, involve the use of the $\mathcal{S} W W$ and $\mathcal{S} Z Z$ vertices in eq. (2.2) implemented in MadGraph [5] using FeynRules [6]. This is correct in either of the following two situations: (i) the origin of the vertices is local physics, originating in scales $\gg M$, such as in the dilaton case in section 3.1.4; in such a case the vertices can be interpreted as a unitary-gauge Lagrangian couplings and be used off shell; or (ii) the production process is dominated by nearly on-shell $W, Z$ bosons (the same prerequisite under which the equivalent-boson approximation $[7,8]$ is justified).
} 


\begin{tabular}{|cccccccc|}
\hline$\sqrt{s}$ & {$[\mathrm{pb}]$} & $g g$ & $u \bar{u}$ & $d \bar{d}$ & $s \bar{s}$ & $c \bar{c}$ & $b \bar{b}$ \\
\hline 13 & $x_{\mathcal{S}}^{13, p}$ & $7.5 \cdot 10^{-3}$ & 250 & 150 & 14 & 9.8 & 4.4 \\
8 & $x_{\mathcal{S}}^{8, p}$ & $1.7 \cdot 10^{-3}$ & 95 & 57 & 3.7 & 2.3 & 0.96 \\
\hline $13 / 8$ & $r_{p}$ & 4.4 & 2.6 & 2.7 & 3.9 & 4.2 & 4.6 \\
\hline
\end{tabular}

\begin{tabular}{|cccccccc|}
\hline$\sqrt{s}$ & {$[\mathrm{pb}]$} & $W W$ & $\widehat{W W}$ & $Z Z$ & $\widehat{Z Z}$ & $Z \gamma$ & $\gamma \gamma$ \\
\hline 13 & $x_{\mathcal{S}}^{13, p}$ & $2.7 \cdot 10^{-6}$ & 0.30 & $8.7 \cdot 10^{-7}$ & $8.3 \cdot 10^{-2}$ & $7.8 \cdot 10^{-7}$ & $1.4 \cdot 10^{-5}$ \\
8 & $x_{\mathcal{S}}^{8, p}$ & $6.5 \cdot 10^{-7}$ & $6.9 \cdot 10^{-2}$ & $2.1 \cdot 10^{-7}$ & $1.9 \cdot 10^{-2}$ & $2.1 \cdot 10^{-7}$ & $4.7 \cdot 10^{-6}$ \\
\hline $13 / 8$ & $r_{p}$ & 4.1 & 4.3 & 4.2 & 4.2 & 3.7 & 2.9 \\
\hline
\end{tabular}

Table 2. Leading-order production cross sections for a resonance with $M=750 \mathrm{GeV}$ for couplings $c_{p}=1$, at the $13 \mathrm{TeV}$ and $8 \mathrm{TeV} \mathrm{LHC}$, and their ratio, $r_{p}$. We have used the leading-order PDF set NN23L01 [9] for the predictions of production via $g g, q \bar{q}, W W, \widehat{W W}, Z Z$ and $\widehat{Z Z}$. For $Z \gamma$ initiated production we use the CTEQ14QED PDF set [10] with photon PDF, while for $\gamma \gamma$ fusion we use the results of ref. [11], which also discusses the validity of $\gamma \gamma$ fusion results obtained with various PDF sets. For the $g g$ and $q \bar{q}$ modes, the process is $p p \rightarrow \mathcal{S}$. For $W W, \widehat{W W}, Z Z$ and $\widehat{Z Z}$, both VBF $(p p \rightarrow \mathcal{S}+j j)$ and associated production $(p p \rightarrow \mathcal{S}+W / Z)$ contribute. The latter is small for $\widehat{W W}$ and $\widehat{Z Z}$ (approximately $1 \%$ of the inclusive value), but is significant for production via the field-strength $W W$ and $Z Z$ operators (approximately $20 \%$ and $30 \%$ for $W W$ and $Z Z$, respectively, at $13 \mathrm{TeV}$; see also ref. [12]). Finally, for production via $Z \gamma$, the processes $p p \rightarrow \mathcal{S} j j, p p \rightarrow \mathcal{S} Z$, and $p p \rightarrow \mathcal{S} \gamma$ contribute with relative weights $94 \%, 2.6 \%$, and $3.6 \%$, respectively.

section and the diphoton branching fraction in this limit, this bound is independent of $\Gamma$. We hence have the following model-independent lower bounds on $c_{\gamma}$ :

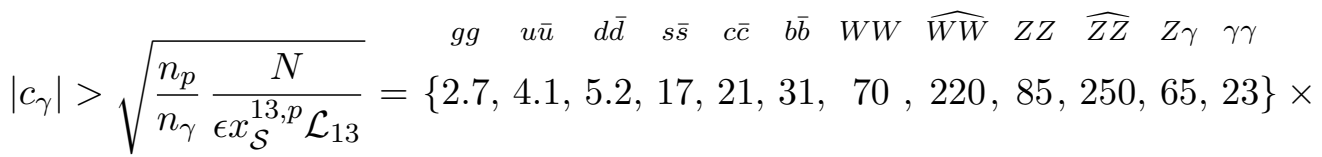

$$
\begin{aligned}
& \times \sqrt{\left(\frac{N}{20}\right)\left(\frac{5.8 \mathrm{fb}^{-1}}{\mathcal{L}_{13}}\right)} .
\end{aligned}
$$

If, as it often does, a single production mode dominates in a concrete model, eq. (2.10) can be directly used to identify how large $c_{\gamma}$ needs to be. For production via $\gamma \gamma$ fusion, saturating the lower bound determines in addition the width to be about $75 \mathrm{MeV}$. In the case where several initial states contribute, a conservative lower bound is given by

$$
\left|c_{\gamma}\right|>\sqrt{\frac{n_{g}}{n_{\gamma}} \frac{N}{\epsilon x_{\mathcal{S}}^{13, g} \mathcal{L}_{13}}}=2.7 \times \sqrt{\left(\frac{N}{20}\right)\left(\frac{5.8 \mathrm{fb}^{-1}}{\mathcal{L}_{13}}\right)} .
$$

Importantly, eqs. (2.8) and (2.9) imply that photon fusion accounts for the entire excess once $\left|c_{\gamma}\right| \sim 110$, or less if the width is below $45 \mathrm{GeV}$. It then follows from eq. (2.10) that production via the couplings $\widehat{c_{W}}$ and $\widehat{c_{Z}}$ can never be an important production mechanism, so we disregard this possibility henceforth. (See also the discussion in ref. [13].) 


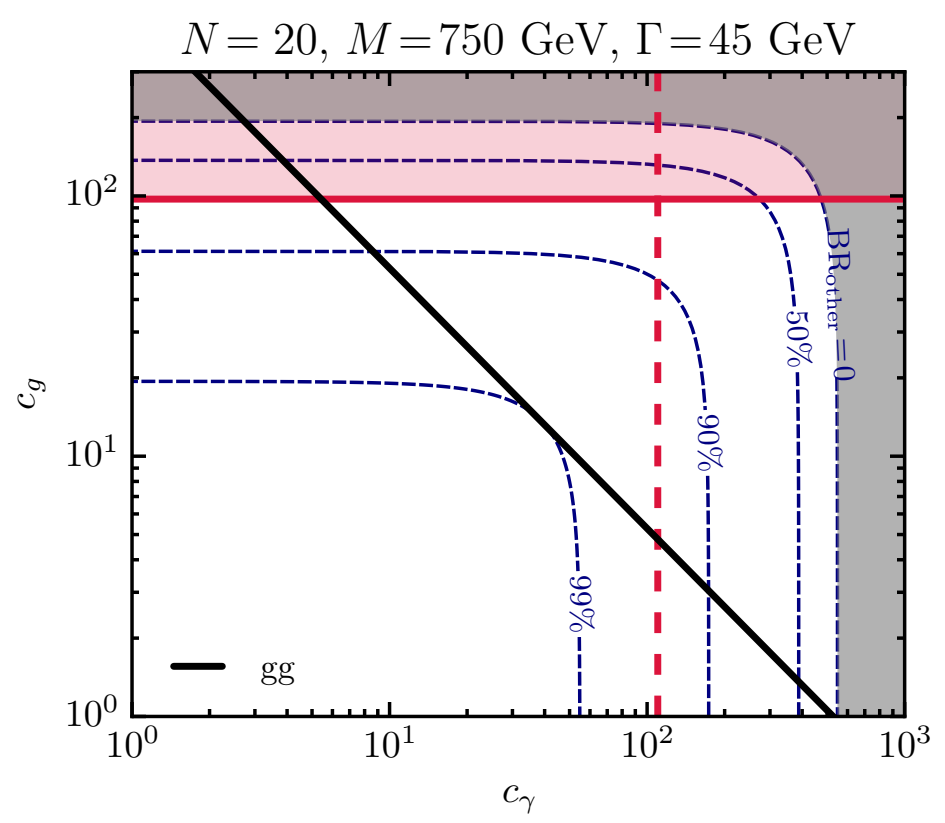

Figure 1. The black line corresponds to $N=20$ signal events in the diphoton analyses for $M=750 \mathrm{GeV}$ and $\Gamma=45 \mathrm{GeV}$ when the resonance is produced from $g g$. Blue dashed lines are contours of fixed branching ratio to modes other than $\gamma \gamma$ or $g g$. The red-shaded area above the thick horizontal line is excluded by dijet resonance searches [14] due to decays to $g g$ alone. The shaded gray region corresponds to values of $c_{g}, c_{\gamma}$ that produce a width larger than $45 \mathrm{GeV}$. The vertical dashed red line indicates the $c_{\gamma}$ value for which photon fusion alone would account for all signal events even for $\Gamma=45 \mathrm{GeV}$, thus ruling out the region of larger $c_{\gamma}$ values.

In figures 1 and 2, we plot the general relation between $\left|c_{\gamma}\right|$ and $\left|c_{p}\right|$ for the case of $N=20$ excess events, switching on one production channel at a time. The mass and total width are fixed at 750 and $45 \mathrm{GeV}$, respectively. The partial widths to diphotons, $\Gamma_{\gamma \gamma}$, and to the production mode, $\Gamma_{p}$, are assumed to be supplemented by decays to other possible final states, $\Gamma_{\text {other }}$, to make up the total width:

$$
\Gamma_{\text {other }} \equiv \Gamma-\Gamma_{\gamma \gamma}-\Gamma_{p}
$$

Contours of fixed $\mathrm{BR}_{\mathrm{other}} \equiv \Gamma_{\mathrm{other}} / \Gamma$ are shown in dashed blue. From the left panels of the figures it is evident that for a given $\mathrm{BR}_{\text {other }}$ there exist two solutions, one with small and another with large $c_{\gamma}$. However, this second solution is generally incompatible with the upper limit $\left|c_{\gamma}\right| \lesssim 110$, unless $\mathrm{BR}_{\text {other }}$ is close to $100 \%$. The gray-shaded regions correspond to values of $c_{p}$ and $c_{\gamma}$ for which the total width is larger than $45 \mathrm{GeV}$. Horizontal red lines and the corresponding red-shaded regions indicate the parameter space excluded by $8 \mathrm{TeV}$ dijet searches. We discuss them in the next subsection.

\subsection{Interplay with previous LHC searches}

Important additional information about the properties of the new particle can be obtained based on the non-observation of any of its decays in Run 1 of the LHC, in particular in the $20 \mathrm{fb}^{-1}$ of data collected at $\sqrt{s}=8 \mathrm{TeV}$. 


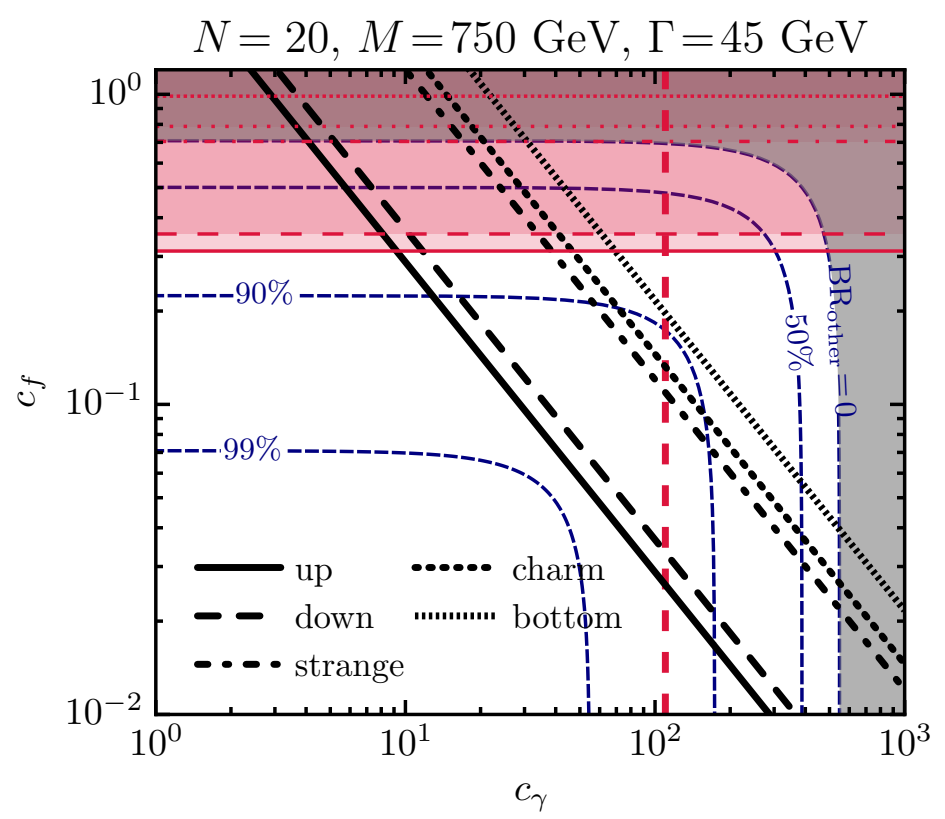

Figure 2. Black lines correspond to $N=20$ signal events in the diphoton analyses for $M=750 \mathrm{GeV}$ and $\Gamma=45 \mathrm{GeV}$. Different dashing styles indicate the various production modes, $u \bar{u}, d \bar{d}, s \bar{s}, c \bar{c}$, and $b \bar{b}$. Blue dashed lines are contours of fixed branching ratio to modes other than $\gamma \gamma$ or the production mode. The red-shaded areas above the various horizontal lines, with dashing styles corresponding to the production modes, are excluded by dijet resonance searches [14] due to decays to the production mode alone. The vertical dashed red line indicates the $c_{\gamma}$ value for which photon fusion alone would account for all signal events even for $\Gamma=45 \mathrm{GeV}$, thus ruling out the region of larger $c_{\gamma}$ values.

We first consider limits from the diphoton resonance searches. The most relevant limit for the broad resonance hypothesis preferred by the ATLAS excess, $\Gamma / M \approx 6 \%$, is the CMS 95\% CL limit

$$
\sigma_{8} \times \mathrm{BR}_{\gamma \gamma} \lesssim 2.5 \mathrm{fb}
$$

which was derived for scalar resonances with $\Gamma / M=10 \%$ [15]. For a narrow resonance, which might be preferred by the CMS data, the same search sets the limit

$$
\sigma_{8} \times \mathrm{BR}_{\gamma \gamma} \lesssim 1.3 \mathrm{fb}
$$

Somewhat weaker limits, of 2.5 and $2.0 \mathrm{fb}$, were obtained by ATLAS [16] and CMS [17], respectively, for RS gravitons with $k=0.1$, which are also narrow.

The compatibility of the observed excesses with the $8 \mathrm{TeV}$ diphoton searches depends primarily on the parton luminosity ratio, $r_{p}$, listed in table $2,{ }^{3}$ since the selection efficiencies

\footnotetext{
${ }^{3}$ More precisely, $r_{p}$ is the cross sections ratio. For VBF and associated production, it cannot be approximated by the parton luminosity ratio at $\sqrt{\hat{s}}=750 \mathrm{GeV}$ (as was done in some of the recent papers that claimed $r_{\mathrm{VBF}} \approx 2.5$ ) since in most events $\sqrt{\hat{s}}$ is significantly higher than $750 \mathrm{GeV}$ because of the two forward jets or the additional electroweak boson.
} 


\begin{tabular}{|c|c|c|c|c|c|c|c|c|c|c|c|}
\hline \multicolumn{2}{|c|}{ decay mode $i \rightarrow$} & $g g$ & $q \bar{q}$ & $t \bar{t}$ & $W W$ & $Z Z$ & $h h$ & $Z h$ & $\tau \tau$ & $Z_{\gamma}$ & $e e+\mu \mu$ \\
\hline \multirow{2}{*}{\multicolumn{2}{|c|}{$\left(\sigma_{8} \times \mathrm{BR}_{i}\right)^{\max }[\mathrm{fb}]$}} & 4000 & 1800 & 500 & 60 & 60 & 50 & 17 & 12 & 8 & 2.4 \\
\hline & & {$[14]$} & {$[14]$} & [18] & [19] & {$[20]$} & {$[21]$} & {$[22]$} & [23] & [24] & {$[25]$} \\
\hline \multirow[t]{4}{*}{ production $p=$} & $g g$ & 2600 & 1200 & 320 & 38 & 38 & 32 & 11 & 7.7 & 5.1 & 1.5 \\
\hline & $u \bar{u}$ & 1500 & 690 & 190 & 23 & 23 & 19 & 6.5 & 4.6 & 3.1 & 0.92 \\
\hline & $d \bar{d}$ & 1600 & 700 & 200 & 23 & 23 & 20 & 6.7 & 4.7 & 3.1 & 0.94 \\
\hline & $s \bar{s}$ & 2300 & 1000 & 280 & 34 & 34 & 28 & 9.6 & 6.8 & 4.5 & 1.4 \\
\hline \multirow{6}{*}{$\left(\frac{\mathrm{BR}_{i}}{\mathrm{BR}_{\gamma \gamma}}\right)^{\max }$} & $c \bar{c}$ & 2400 & 1100 & 300 & 36 & 36 & 30 & 10 & 7.3 & 4.8 & 1.5 \\
\hline & $b \bar{b}$ & 2700 & 1200 & 340 & 40 & 40 & 34 & 11 & 8.1 & 5.4 & 1.6 \\
\hline & $W W$ & 2400 & 1100 & 300 & 35 & 35 & 30 & 10 & 7.1 & 4.7 & 1.4 \\
\hline & $Z Z$ & 2400 & 1100 & 310 & 37 & 37 & 31 & 10 & 7.3 & 4.9 & 1.5 \\
\hline & $Z_{\gamma}$ & 2200 & 980 & 270 & 33 & 33 & 27 & 9.2 & 6.5 & 4.3 & 1.3 \\
\hline & $\gamma \gamma$ & 1700 & 760 & 210 & 25 & 25 & 21 & 7.1 & 5.0 & 3.4 & 1.0 \\
\hline
\end{tabular}

Table 3. Top: bounds on $750 \mathrm{GeV}$ resonances from $8 \mathrm{TeV}$ LHC searches. Bottom: derived bounds on ratios of branching fractions for different production channel assumptions. For $g g$ production, bounds on the branching fraction to $q \bar{q}$ are even tighter than indicated, since decays to $g g$ will necessarily also be present and enter the dijet searches. The same applies to branching fractions to $g g$ when the production is from quarks.

of the searches are similar. The ATLAS+CMS excess, eq. (2.1), translates to

$$
\sigma_{8} \times \mathrm{BR}_{\gamma \gamma}=\frac{\sigma_{13} \times \mathrm{BR}_{\gamma \gamma}}{r_{p}} \approx\left(\frac{N}{20}\right) \times\{1.6,2.6,2.6,1.8,1.7,1.5,1.7,1.6,1.8,2.4\} \mathrm{fb} .
$$

We see that $N=20$ excess events at $13 \mathrm{TeV}$ are borderline compatible with the $8 \mathrm{TeV}$ analyses, especially if the resonance is broad. The $g g$, heavy-quark and electroweak-boson production modes are somewhat favoured in this respect because their cross sections increase more rapidly with $\sqrt{s}$.

The ATLAS and CMS collaborations performed searches for resonant signals in many other final states as well. In table 3 we list the various two-body final states relevant to a neutral color-singlet spin-0 particle, and the corresponding 95\% C.L. exclusion limits, $\left(\sigma_{8} \times \mathrm{BR}_{i}\right)^{\mathrm{max}}$, from the $8 \mathrm{TeV}$ searches. Searches for dijet resonances that employ $b$ tagging, and would have enhanced sensitivity to $b \bar{b}$ final states, only address resonances heavier than $1 \mathrm{TeV}[26,27]$, but the limits from $q \bar{q}$ searches still apply to $b \bar{b}$. The recent $13 \mathrm{TeV}$ dijet searches $[28,29]$ do not cover the mass range around $750 \mathrm{GeV}$ at all, due to triggering limitations. We also note that the limits quoted in table 3 are approximate. In general, they do have some dependence on the width of the resonance, its spin, etc.

Table 3 also lists the resulting constraints on the ratios of branching fractions of the particle, for different production channel assumptions. They are computed as

$$
\left(\frac{\mathrm{BR}_{i}}{\mathrm{BR}_{\gamma \gamma}}\right)^{\max }=r_{p} \frac{\left(\sigma_{8} \times \mathrm{BR}_{i}\right)^{\max }}{\sigma_{13} \times \mathrm{BR}_{\gamma \gamma}},
$$

where we use eq. (2.1) and the cross section ratios $r_{p}$ from table 2. 
There is always a constraint from decays to dijets or dibosons since we take the resonance to couple to either $g g, q \bar{q}$, or the electroweak gauge bosons, for production. Also, the production cross section needs to be relatively large to accommodate the excess without too large $c_{\gamma}$, so limits on dijet or diboson resonances may restrict part of the parameter space of a concrete realisation. For the case in which a single production channel dominates, we obtain upper limits on $\mathrm{BR}_{p}$ and $c_{p}$ by saturating the corresponding dijet or diboson bounds:

$$
\begin{aligned}
& \mathrm{BR}_{p}<\sqrt{n_{p} \frac{M}{\Gamma} \frac{\left(\sigma_{8} \times \mathrm{BR}_{p}\right)^{\mathrm{max}}}{x_{\mathcal{S}}^{8, p}}} \approx\{25,19,25,99,120,190, \quad 76,94,25,4.2\} \% \times\left(\frac{45 \mathrm{GeV}}{\Gamma}\right)^{1 / 2},
\end{aligned}
$$

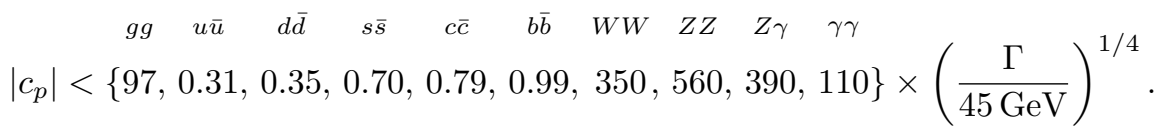

The dijet-excluded regions in the $c_{p}-c_{\gamma}$ planes of figures 1 and 2 are the red-shaded areas.

By combining eq. (2.17) with eqs. (2.4) and (2.8), we obtain a second lower bound on $\mathrm{BR}_{\gamma \gamma}$ and $c_{\gamma}$,

$$
\begin{aligned}
& \begin{array}{llllllllll}
g g & u \bar{u} & d \bar{d} & s \bar{s} & c \bar{c} & b \bar{b} & W W & Z Z & Z \gamma & \gamma \gamma
\end{array} \\
& \mathrm{BR}_{\gamma \gamma}>\{0.98,2.8,3.5,9.7,11,16,210,260,570,400\} \times 10^{-4} \times\left(\frac{N}{20}\right)\left(\frac{5.8 \mathrm{fb}^{-1}}{\mathcal{L}_{13}}\right)\left(\frac{45 \mathrm{GeV}}{\Gamma}\right)^{1 / 2}, \\
& \begin{array}{cccccccccc}
g g & u \bar{u} & d \bar{d} & s \bar{s} & c \bar{c} & b \bar{b} & W W & Z Z & Z \gamma & \gamma \gamma \\
\left|c_{\gamma}\right|>\{5.4,9.2,10,17,18,22, & 80 & 88,130,110\} \times \sqrt{\left(\frac{N}{20}\right)\left(\frac{5.8 \mathrm{fb}^{-1}}{\mathcal{L}_{13}}\right)}\left(\frac{\Gamma}{45 \mathrm{GeV}}\right)^{1 / 4} .
\end{array}
\end{aligned}
$$

Depending on the width and the production mechanism, these bounds can be stronger or weaker than those in eqs. (2.7) and (2.10). Some comments are in order regarding the case of photon fusion dominance. In this case, eqs. (2.17) and (2.18) fix, for nominal width and signal strength, $\left|c_{\gamma}\right| \approx 110$. This is because here we impose an upper bound of $2.5 \mathrm{fb}$ for the $8 \mathrm{TeV}$ diphoton signal, which essentially agrees with the predicted $8 \mathrm{TeV}$ signal, for nominal width and number of excess events. For the same reason, this value agrees with the one previously obtained based on saturating the $13 \mathrm{TeV}$ signal with a single diphoton coupling.

Figures 3 and 4 show, for $\Gamma=45 \mathrm{GeV}$ and $1 \mathrm{GeV}$, respectively, the required branching fraction $\mathrm{BR}_{\text {other }}$ to modes other than the production mode and $\gamma \gamma$ as a function of the branching fraction of the production mode, $\mathrm{BR}_{p}$. The black lines correspond to $N=20$ signal events in the $13 \mathrm{TeV}$ diphoton analyses. These plots highlight the importance of $\mathrm{BR}_{\mathrm{other}}$, which in most of the viable parameter space is the dominant branching fraction if the width $\Gamma$ is large. In blue lines, it is also shown to what extent $\mathrm{BR}_{\text {other }}$ can be attributed to various decays into Standard Model particles, in view of the $8 \mathrm{TeV}$ LHC bounds on such decays. For example, if apart from the decays to the production mode and $\gamma \gamma$ the resonance can decay only to $t \bar{t}$, the region above the corresponding blue line is excluded. The solid blue lines labeled "all" correspond to saturating all the two-body final states listed in table 3, with the band interpolating between lines that use the $g g$ and the $q \bar{q}$ dijet bounds. The band is needed since the maximal possible $\mathrm{BR}_{\text {other }}$ is generally achieved for a mixture of $g g$ and $q \bar{q}$ decays. Indeed, for a fixed $\mathrm{BR}_{g g}$, one can add decays to quarks. For a fixed $\mathrm{BR}_{q \bar{q}}$ (for a given flavor $q$ ), one can add decays to either gluons or other quark flavors, but gluons are preferable since they are less constrained. It is reasonable to expect the bound 


\section{$\Gamma=45 \mathrm{GeV}$}
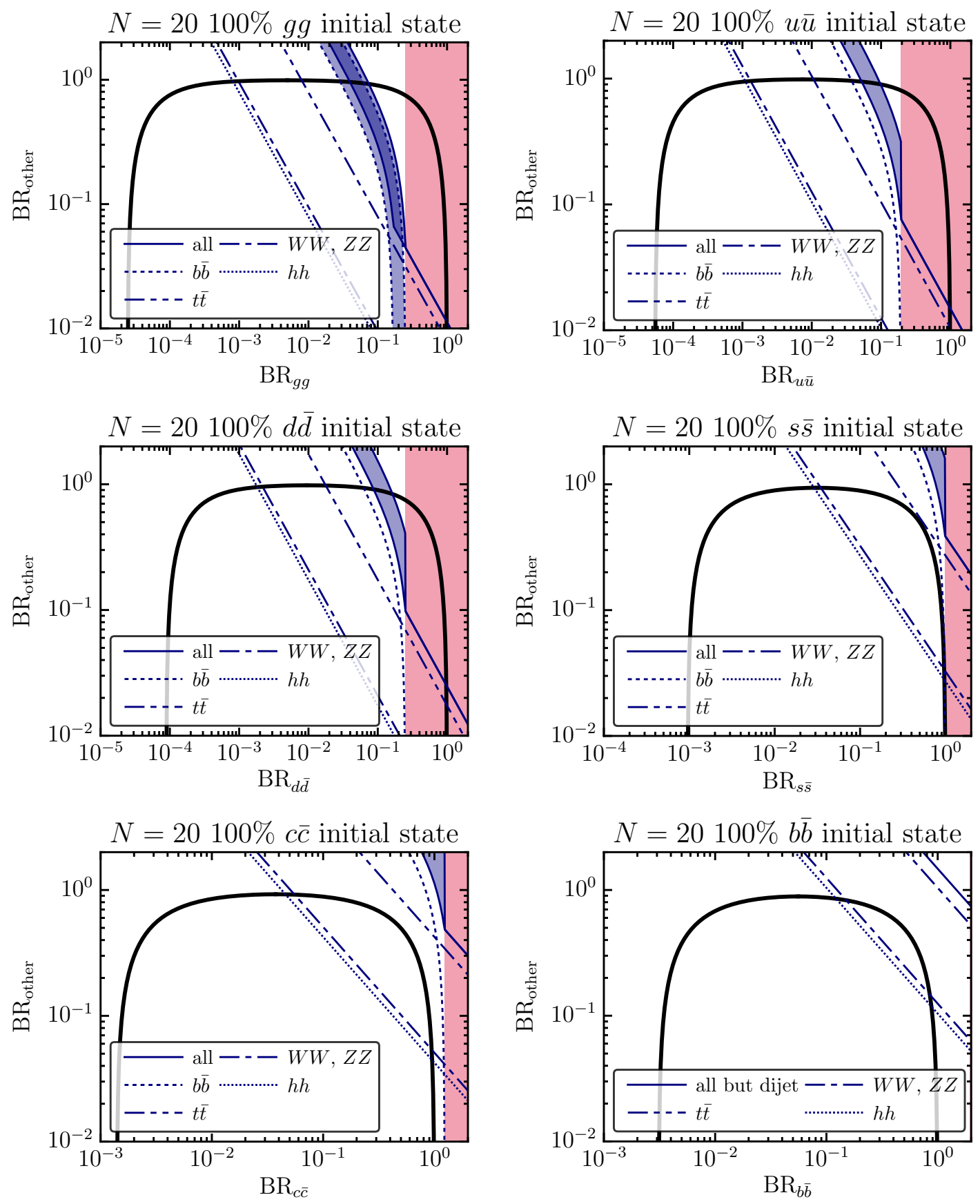

Figure 3. The required branching fraction into modes other than the production mode and $\gamma \gamma$, $\mathrm{BR}_{\text {other }}$, as a function of the production mode branching fraction, for $N=20$ and $\Gamma=45 \mathrm{GeV}$. Different plots correspond to different production mechanisms. Red regions are excluded by $8 \mathrm{TeV}$ dijet resonance searches. Thin lines described in the legend show the maximal branching fractions allowed by $8 \mathrm{TeV}$ searches into final states from table 3. The label "all" refers to the bound on the sum of all the final states from the table. For mixed dijet final states $(g g+q \bar{q})$, we show bands extending between curves obtained using the $g g$ and the $q \bar{q}$ dijet constraint. 

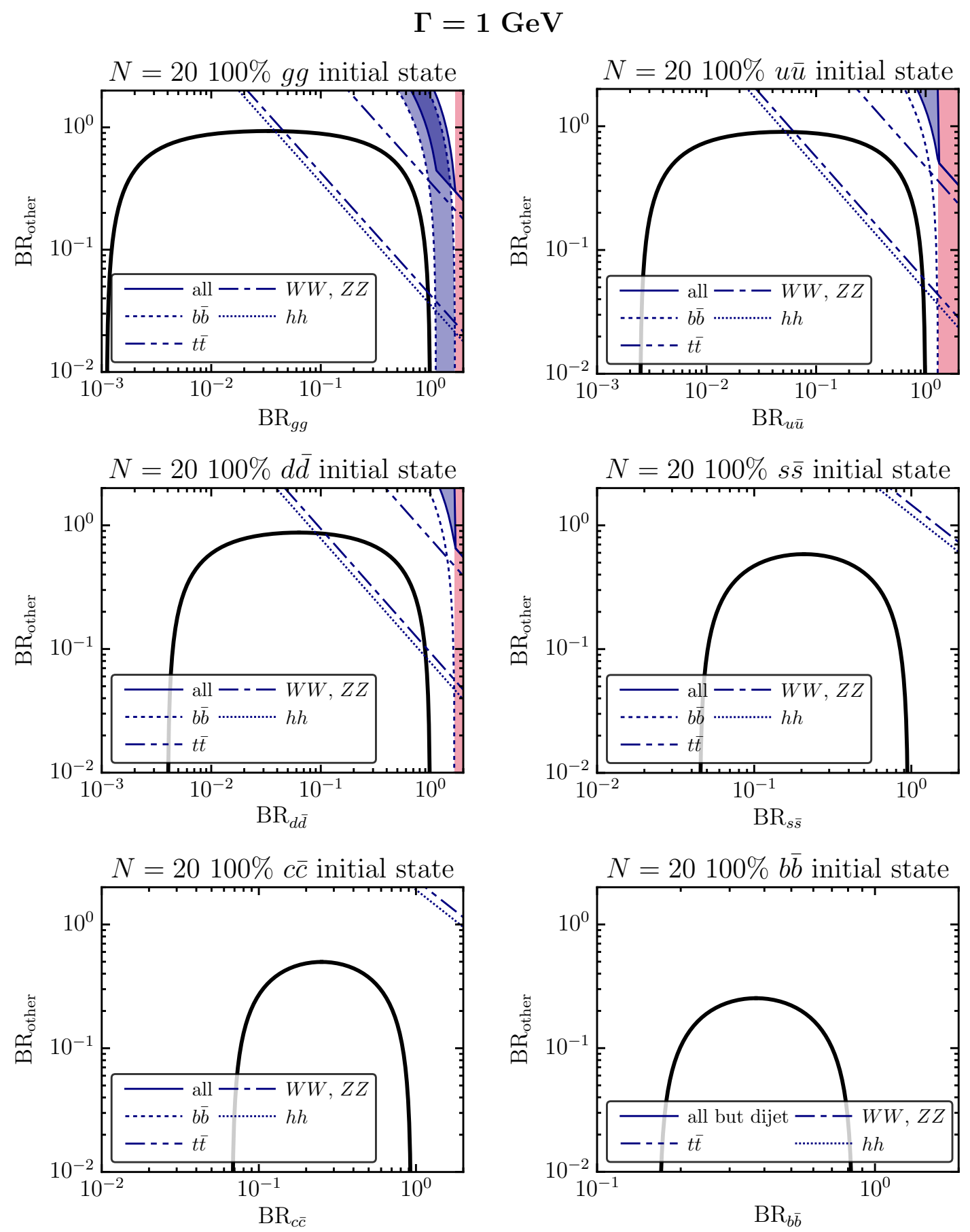

Figure 4. The required branching fraction into modes other than the production mode and $\gamma \gamma$, $\mathrm{BR}_{\text {other }}$, as a function of the production mode branching fraction, for $N=20$ and $\Gamma=1 \mathrm{GeV}$. Different plots correspond to different production mechanisms. Red regions are excluded by $8 \mathrm{TeV}$ dijet resonance searches. Thin lines described in the legend show the maximal branching fractions allowed by $8 \mathrm{TeV}$ searches into final states from table 3. The label "all" refers to the bound on the sum of all the final states from the table. For mixed dijet final states $(g g+q \bar{q})$, we show bands extending between curves obtained using the $g g$ and the $q \bar{q}$ dijet constraint. 
on such mixed final states to lie somewhere within the band. The same discussion applies to the $b \bar{b}$ band in the fixed $\mathrm{BR}_{g g}$ case.

We see that when the diphoton signal is achieved by a large coupling to gluons/quarks and a small coupling to photons (right-hand side of the plots), it may be difficult to obtain $\Gamma=45 \mathrm{GeV}$ with decays to $\mathrm{SM}$ particles alone (if we neglect the possibility of large branching fractions to $\nu \bar{\nu}$ or multibody final states). On the other hand, in the case of a small coupling to gluons/quarks and a large coupling to photons (left side of the plots) there is no such limitation.

\section{Models}

We now turn to discuss concrete models. First, in section 3.1, we discuss interpretations of the resonance as a scalar that is a singlet under the SM gauge group. Next, in section 3.2, we consider the possibility of an $\mathrm{SU}(2)_{L}$ doublet. Finally, in section 3.3 we study whether the resonance can be a heavy Higgs of the MSSM.

\subsection{SM-singlet scalar}

The possible interactions of a real singlet scalar with the SM fields, up to dimension-five terms, are

$$
\begin{aligned}
\mathcal{L}_{\text {singlet }}= & \left(\mu \Phi+\kappa_{H 1} \Phi^{2}\right) H^{\dagger} H \\
& +\frac{\Phi}{f}\left(\kappa_{g} \frac{\alpha_{s}}{8 \pi} G_{\mu \nu} G^{\mu \nu}+\kappa_{Y} \frac{\alpha_{1}}{8 \pi} B_{\mu \nu} B^{\mu \nu}+\kappa_{W} \frac{\alpha_{2}}{8 \pi} W_{\mu \nu} W^{\mu \nu}+\kappa_{H 2}\left|D_{\mu} H\right|^{2}+\kappa_{H 3}|H|^{4}\right) \\
& -\frac{\Phi}{f}\left(Y_{i j}^{d} H \overline{Q_{i}} d_{j}+Y_{i j}^{u} \tilde{H} \overline{Q_{i}} u_{j}+Y_{i j}^{e} H \overline{L_{i}} e_{j}+\text { h.c. }\right) .
\end{aligned}
$$

We first discuss the renormalizable scenario in which only the terms on the first line are present. Next we consider a, still renormalizable, model where the diphoton and digluon couplings $c_{g}$ and $c_{\gamma}$ are generated by additional vectorlike fermions. We also analyze the pseudoscalar case, where the possible interactions differ in several important ways from eq. (3.1), as we will discuss. We then turn to scenarios where the nonrenormalizable couplings on the second and third line are present, generated by physics above the scale $M$ and resulting in "local" contributions to the couplings $c_{g}$ and $c_{\gamma}$. We consider the dilaton scenario of ref. [30] (except that the dilaton is in addition to the Higgs) in which the $\kappa_{g, Y, W}$ are related to the $\beta$ functions in the low-energy effective theory. Finally we discuss the possibility of production of the resonance by quarks due to the presence of the couplings in the last line of eq. (3.1).

\subsubsection{Renormalizable model}

We consider the case with only the renormalizable couplings in eq. (3.1). The $\mu$ term induces mixing of $\Phi$ with the SM-like Higgs field, and we obtain two mass eigenstates, $\mathcal{S}$ and the observed $125 \mathrm{GeV}$ Higgs $h$. This results in $\mathcal{S}$ having tree-level couplings proportional to those of the SM Higgs but suppressed by a universal factor,

$$
g_{i}^{\mathcal{S}}=s_{\alpha} g_{i}^{h_{\mathrm{SM}}}
$$


where $s_{\alpha} \equiv \sin \alpha, \alpha$ being the mixing angle. This mixing also modifies the couplings of the observed $125 \mathrm{GeV}$ Higgs boson with respect to what they would be in the SM. The modified couplings are scaled by $\cos \alpha$ with respect to their SM values. We must thus have $s_{\alpha} \lesssim 0.2$ to ensure that these modifications are compatible with Higgs and particularly electroweak precision measurements [31]. The coupling to the light quarks is thus negligible, therefore the production must be gluonic. At one-loop level, SM particles generate an effective $c_{g}$ and $c_{\gamma}$. To get the largest possible $c_{g}$ and $c_{\gamma}$ we take $s_{\alpha}=0.2$ and obtain using the expressions for the top and $W$-loop contributions in ref. [32]

$$
\left|c_{g}\right|=1.6, \quad\left|c_{\gamma}\right|=0.09
$$

If we assume a $45 \mathrm{GeV}$ width, we need $\left|c_{g} c_{\gamma}\right| \approx 530$ to accommodate the excess (cf. eq. (2.8)), so these numbers are far too small. Even if we allow for a smaller width, they still do not satisfy the bound $\left|c_{\gamma}\right| \gtrsim 2.7$ from eq. (2.10).

Clearly we need large contributions from BSM states to $c_{\gamma}$, and either $c_{g}$ or the couplings $c_{f}$ to quarks, in eq. (2.2), to explain the size of the excess.

\subsubsection{Boosting $c_{\gamma}, c_{g}$ with new vectorlike fermions}

To investigate whether new colored and charged particles can generate large enough $c_{g, \gamma}$, we consider the minimal case of an additional vectorlike fermion, a triplet under QCD with electric charge $Q_{f}$, that couples to $\Phi$ as

$$
\mathcal{L}=-y_{Q} \Phi \bar{Q} Q-m_{Q} \bar{Q} Q
$$

The fermion loop generates $c_{g, \gamma}$. Any mixing of $\Phi$ with the Higgs doublet would dilute the vectorlike loop contributions (which would be generally larger than the SM loop contributions) to the diphoton and digluon couplings of the mass eigenstate $\mathcal{S}$. Thus, we assume that the mixing, which is in any case constrained to be small, is negligible and the mass eigenstate is $\mathcal{S}=\Phi$.

The fermion $Q$ contributes [32]

$$
\begin{aligned}
& c_{g}=g_{s}^{2} y_{Q} \tilde{A}_{1 / 2}\left(\tau_{Q}\right), \\
& c_{\gamma}=2 N_{c} Q_{f}^{2} e^{2} y_{Q} \tilde{A}_{1 / 2}\left(\tau_{Q}\right),
\end{aligned}
$$

where $N_{c}=3$ is the number of color states, $\tau_{Q}=M^{2} /\left(4 m_{Q}^{2}\right)$ and

$$
\tilde{A}_{1 / 2}(\tau)=2 \tau^{1 / 2} A_{1 / 2}(\tau)=4 \tau^{-3 / 2}[\tau+(\tau-1) f(\tau)]
$$

where

$$
f(\tau)= \begin{cases}\arcsin ^{2} \sqrt{\tau} & \tau \leq 1 \\ -\frac{1}{4}\left[\ln \frac{1+\sqrt{1-\tau^{-1}}}{1-\sqrt{1-\tau^{-1}}}-i \pi\right]^{2} & \tau>1 .\end{cases}
$$

For $m_{Q}<M / 2$ we obtain the constraint $y_{Q} \lesssim 0.7$ by requiring $\Gamma(\mathcal{S} \rightarrow Q \bar{Q}) \lesssim 45 \mathrm{GeV}$. This would not allow generating sufficiently large values of $c_{g, \gamma}$. We thus take $m_{Q}>M / 2$. 

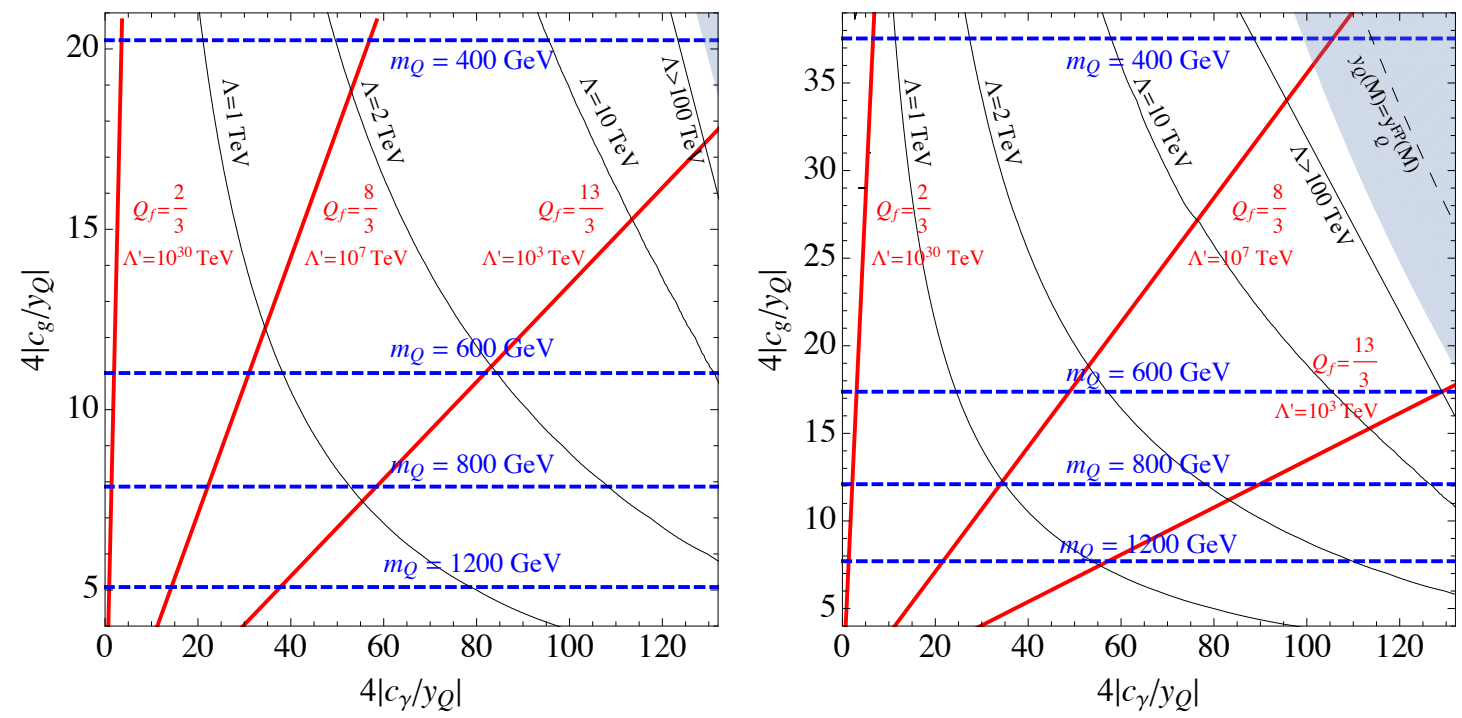

Figure 5. For a SM-singlet scalar (left) or pseudoscalar (right), contributions of a vectorlike colortriplet fermion with mass $m_{Q}$, charge $Q_{f}$ and Yukawa coupling $y_{Q}$ to the photonic, $c_{\gamma}$, and gluonic, $c_{g}$, couplings, scaled by $4 / y_{Q}$. Black lines are contours of the scale $\Lambda$ at which the theory becomes strongly coupled if the value of $y_{Q}$ at the scale $M$ is fixed by requiring the correct signal size $(N=20)$, assuming $\Gamma=45 \mathrm{GeV}$. The diagonal solid thick red lines stand for different values of $Q_{f}$; on each line, the corresponding scale for the Landau pole for the hypercharge interaction, $\Lambda^{\prime}$ is shown. The horizontal blue dashed lines refer to different values of $m_{Q}$. We shade the regions where $y_{Q}^{2}(M)$ is within $20 \%$ of $\left[y_{Q}^{\mathrm{FP}}(M)\right]^{2}$ (see eq. (3.12)). The dashed black line is the contour where $y_{Q}(M)=y_{Q}^{\mathrm{FP}}(M)$.

In figure 5 (left), we show the resulting $c_{g, \gamma}$ for a range of $m_{Q}$ and $Q_{f}$. The values of $c_{g, \gamma}$ for $y_{Q}=4$ can be directly read off from the plot, and one can easily find them for other $y_{Q}$ values by keeping in mind that $c_{g, \gamma}$ scale linearly with $y_{Q}$.

The same fermions will generically also generate couplings to $W^{+} W^{-}, Z Z$ and $Z \gamma$. While a detailed study of the various possibilities is beyond the scope of this paper, we note that the bounds from table 3 are easily satisfied if, for example, the fermions are $\mathrm{SU}(2)_{L}$ singlets. This is because they only contribute to the $\kappa_{Y}$ coupling from eq. (3.1), but not to $\kappa_{W}$, so one has $\mathrm{BR}_{Z \gamma} / \mathrm{BR}_{\gamma \gamma}=2 \tan ^{2} \theta_{W} \approx 0.6, \mathrm{BR}_{Z Z} / \mathrm{BR}_{\gamma \gamma}=\tan ^{4} \theta_{W} \approx 0.1$, and no contribution to $W^{+} W^{-}$.

Since the Yukawa couplings $y_{Q}$ needed to reproduce the diphoton signal are relatively large, it is important to check to what extent the theory remains perturbative in the UV. We first consider the case in which we assume a $45 \mathrm{GeV}$ width for the resonance. In some regions of the parameter space, this implies a low cut-off for the theory at the scale at which $y_{Q}$ becomes strongly coupled. For $n_{f}$ color-triplet, $\mathrm{SU}(2)_{L}$-singlet vector-like fermions, the 
RGE are given by ${ }^{4}$

$$
\begin{aligned}
\frac{d y_{Q}}{d \ln \mu} & =\frac{y_{Q}}{16 \pi^{2}}\left(\left(3+6 n_{f}\right) y_{Q}^{2}-6 Q_{f}^{2} g^{\prime 2}-8 g_{s}^{2}\right), \\
\frac{d g^{\prime}}{d \ln \mu} & =\frac{g^{\prime 3}}{16 \pi^{2}}\left(\frac{41}{6}+4 n_{f} Q_{f}^{2}\right) \\
\frac{d g_{s}}{d \ln \mu} & =\frac{g_{s}^{3}}{16 \pi^{2}}\left(-7+\frac{2}{3} n_{f}\right)
\end{aligned}
$$

(see, e.g., ref. [34]); as said above we will only consider the minimal case $n_{f}=1$. We show in figure 5 (left) contours of the scale $\Lambda$ at which the theory becomes strongly coupled, assuming $y_{Q}$ to be just large enough at each point in figure 5 (left) to explain the excess. We take as the strong coupling scale $\Lambda$ the scale where either $\sqrt{N_{c}} y_{Q}$ or (only in some part of the region marked $\Lambda>100 \mathrm{TeV}) \sqrt{N_{c}} Q_{f} g^{\prime}$ becomes $\mathcal{O}(4 \pi)\left(N_{c}=3\right)$.

For the theory to remain weakly coupled above the scale of roughly $10 \mathrm{TeV}$, a rather large value for the electric charge $Q_{f}$ is required, roughly above 3 , for most of the shown parameter space. For a large charge, the negative contribution to the running proportional to $y_{Q} Q_{f}^{2} g^{\prime 2}$ in eq. (3.9) can actually push the cut-off up to $100 \mathrm{TeV}$, as shown in the topright part of figure 5 (left). The RGE of $y_{Q}$ has a perturbative quasi fixed point, which in the one-loop approximation, eq. (3.9), is given by

$$
y_{Q}^{\mathrm{FP}}=\frac{1}{3} \sqrt{8 g_{s}^{2}+6 g^{\prime 2} Q_{f}^{2}} \approx 1.15 \sqrt{1+0.066 Q_{f}^{2}} .
$$

Therefore, for an IR value (at the diphoton resonance mass scale) satisfying $y_{Q}<y_{Q}^{\mathrm{FP}}$, the cutoff of the theory will likely be given by the Landau pole of the hypercharge interaction. It is controlled at high energies by the rather large charge of the vector-like quarks. On the other hand, for $y_{Q}>y_{Q}^{\mathrm{FP}}$, the Yukawa coupling typically grows with energy. In such a case, the cutoff of the theory is set by the Landau pole of $y_{Q}$. We also note that generically, for UV boundary conditions that satisfy $y_{Q}\left(\Lambda_{\mathrm{UV}}\right)>y_{Q}^{\mathrm{FP}}\left(\Lambda_{\mathrm{UV}}\right)$, we expect to have $y_{Q}^{\mathrm{IR}} \sim y_{Q}^{\mathrm{FP}}$. Since the one-loop $\beta$ function for $y_{Q}$ is small in this region, the impact of two-loop contributions may be nonnegligible. To indicate this, the region in which $y_{Q}^{2}(M)$ is within $\pm 20 \%$ of $\left[y_{Q}^{\mathrm{FP}}(M)\right]^{2}$ is shaded.

We note that after rescaling $y_{Q}$ appropriately to explain the signal, the partial width to photons and gluons never exceeds $5 \mathrm{GeV}$ in the region $Q_{f}>2 / 3$, so significant decays to other final states are needed to explain the $45 \mathrm{GeV}$ width. The dijet constraint $\left|c_{g}\right| \lesssim 97$ (see eq. (2.17)) is satisfied in the above region assuming that there are no dijet final states other than $g g$.

We now consider the case in which the resonance is narrow and the width is dominated by decays to $g g$. We then only need a $\left|c_{\gamma}\right| \approx 2.7$ according to eq. (2.10). In figure 6 (left) we show the scale of breakdown of perturbativity assuming the required $y_{Q}$ to obtain $\left|c_{\gamma}\right|=2.7$ from the loop of a vectorlike color-triplet fermion, as a function of its mass, $m_{Q}$. We see that the theory might be perturbative up to high scales even for much smaller electric charges.

\footnotetext{
${ }^{4}$ Note that the couplings $\mu_{3} \mathcal{S}^{3}$ and $\lambda_{S} \mathcal{S}^{4}$ do not alter these RG equations. For a more comprehensive analysis that considers the running of the quartic coupling $\lambda_{S}$, see for instance ref. [33] where it is shown that if the value of $\lambda_{S}$ at the low scale is appropriately chosen, it can remain perturbative up to the Planck scale.
} 

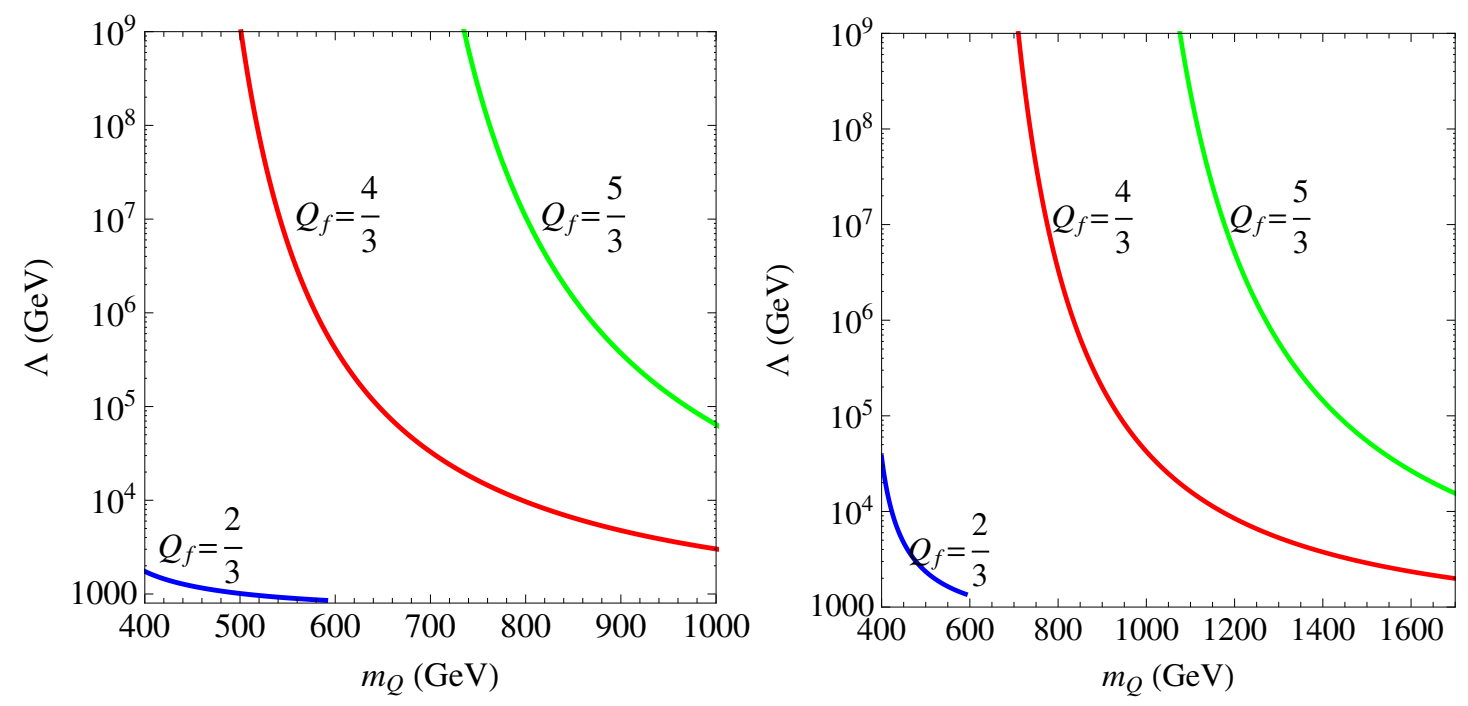

Figure 6. For a narrow-width SM-singlet scalar (left) or pseudoscalar (right), the scale of breakdown of perturbativity as a function of the color-triplet vectorlike fermion mass $m_{Q}$, assuming we take the required $y_{Q}$ at the scale $M$ to explain the excess. For $m_{Q} \lesssim 490 \mathrm{GeV}\left(m_{Q} \lesssim 720 \mathrm{GeV}\right)$ with $Q_{f}=4 / 3\left(Q_{f}=5 / 3\right)$ for the scalar or $m_{Q} \lesssim 700 \mathrm{GeV}\left(m_{Q} \lesssim 1050 \mathrm{GeV}\right)$ with $Q_{f}=4 / 3$ $\left(Q_{f}=5 / 3\right)$ for the pseudoscalar, $y_{Q}(M) \lesssim y_{Q}^{\mathrm{FP}}$ in eq. (3.12) and the $\beta$ function is negative at the scale $M$, so the theory is perturbative.

\subsubsection{A pseudoscalar}

We now consider the case of a pseudoscalar. Unlike the scalar it cannot mix with the SM Higgs and some couplings like those to longitudinal $W$ 's and $Z$ 's in eq. (3.1) are not allowed. The possible interaction terms are

$$
\begin{aligned}
\mathcal{L}= & -\frac{1}{16 \pi^{2}} \frac{1}{4} \frac{c_{B}}{M} \mathcal{S} B^{\mu \nu} \tilde{B}_{\mu \nu}-\frac{1}{16 \pi^{2}} \frac{1}{4} \frac{c_{W}}{M} \mathcal{S} W^{a \mu \nu} \tilde{W}_{\mu \nu}^{a}-\frac{1}{16 \pi^{2}} \frac{1}{4} \frac{c_{g}}{M} \mathcal{S} G^{\mu \nu, a} \tilde{G}_{\mu \nu}^{a} \\
& -\frac{\mathcal{S}}{f}\left(i Y_{i j}^{d} H \overline{Q_{i}} d_{j}+i Y_{i j}^{u} \tilde{H} \overline{Q_{i}} u_{j}+i Y_{i j}^{e} H \overline{L_{i}} e_{j}+\text { h.c. }\right)-y_{Q} \mathcal{S} \bar{Q} i \gamma_{5} Q \\
\supset & -\frac{1}{16 \pi^{2}} \frac{1}{4} \frac{c_{\gamma}}{M} \mathcal{S} F^{\mu \nu} \tilde{F}_{\mu \nu}-\frac{1}{16 \pi^{2}} \frac{1}{4} \frac{c_{g}}{M} \mathcal{S} G^{\mu \nu, a} \tilde{G}_{\mu \nu}^{a}-y_{Q} \mathcal{S} \bar{Q} i \gamma_{5} Q
\end{aligned}
$$

where we have also included a coupling to a vectorlike quark $Q$. A pseudoscalar can appear in composite models as a pseudo-Nambu-Goldstone boson (PNGB) with sizeable couplings to photons and gluons because of anomalies [35], but here we will consider the possibility where $c_{g}$ and $c_{\gamma}$ are generated only from loops of the fermion $Q$. These loop contributions are given by [32]

$$
\begin{aligned}
& c_{g}=2 g_{s}^{2} y_{Q} \tilde{A}_{1 / 2}^{P S}\left(\tau_{Q}\right), \\
& c_{\gamma}=4 N_{c} Q_{f}^{2} e^{2} y_{Q} \tilde{A}_{1 / 2}^{P S}\left(\tau_{Q}\right),
\end{aligned}
$$

where $\tau_{Q}=M^{2} /\left(4 m_{Q}^{2}\right)$ and

$$
\tilde{A}_{1 / 2}^{P S}(\tau)=2 \tau^{1 / 2} A_{1 / 2}^{P S}(\tau)=2 \tau^{-1 / 2} f(\tau),
$$

with $f(\tau)$ defined in eq. (3.8). 
Following the same procedure as in section 3.1.2, we obtain the results shown in the right plots of figures 5 and 6 . They are qualitatively similar to the scalar case, but the theory can be perturbative up to somewhat higher scales for given $Q_{f}$ and $m_{Q}$.

Again, in figure 5 (right) we shade the region in which $y_{Q}^{2}(M)$ is within $20 \%$ of $\left[y_{Q}^{\mathrm{FP}}(M)\right]^{2}$ of eq. (3.12). This is the region where the cut-off can be high but at the same time our one-loop computation may be less reliable. The dashed black line is the contour where $y_{Q}(M)=y_{Q}^{\mathrm{FP}}(M)$.

We thus find that for a $\Gamma \approx 45 \mathrm{GeV}$ singlet resonance (in both the scalar and pseudoscalar cases) the size of the excess suggests strongly coupled physics at a few $\mathrm{TeV}$ unless there are additional new particles around or below the mass of the resonance with large electric charge. For the narrow width case we still need to require additional charged states but the theory can be perturbative with a smaller electric charge. The hints of strongly coupled physics motivate us to examine in more detail a popular strongly coupled scalar candidate, the dilaton.

\subsubsection{The dilaton}

We consider a generalization of the dilaton scenario of ref. [30], taking the full SM, including the Higgs doublet, to be part of a conformal sector (see also [36]). The dilaton is the PNGB of the spontaneously broken scale invariance. The couplings of the dilaton in the electroweak broken phase are given by

$$
\begin{aligned}
\mathcal{L}^{\text {dil }}=\frac{\Phi}{f} & \left(\left(\partial_{\mu} h_{\mathrm{SM}}\right)^{2}+2\left(m_{W}^{2} W^{+} W^{-}+\frac{m_{Z}^{2}}{2} Z^{2}\right)\right. \\
& \left.-\sum_{f} m_{f} \bar{f} f+\frac{\kappa_{g} \alpha_{s}}{8 \pi} G_{\mu \nu} G^{\mu \nu}+\frac{\kappa_{\gamma} \alpha}{8 \pi} F_{\mu \nu} F^{\mu \nu}\right),
\end{aligned}
$$

where the first three terms arise from the operator $2 \Phi\left|D_{\mu} H\right|^{2} / f$. Note that the dilaton also couples to the $W^{ \pm}$and $Z$ field strengths, but these operators have loop-suppressed Wilson coefficients and thus their contribution is subdominant compared to the contribution from $2 \Phi\left|D_{\mu} H\right|^{2} / f$. Furthermore, there will be a mixing term with the SM Higgs, that will arise from the potential term $\Phi H^{\dagger} H$ and possibly also from kinetic mixing, so that finally we obtain two mass eigenstates, $\mathcal{S}$ and the observed $125 \mathrm{GeV}$ Higgs $h$, where

$$
\mathcal{S}=s_{\alpha} h_{\mathrm{SM}}+c_{\alpha} \Phi
$$

and $s_{\alpha}=\mathcal{O}(v / f)$ and $c_{\alpha}=1$ up to $\mathcal{O}\left(v^{2} / f^{2}\right) .{ }^{5}$ We thus have for the couplings to the massive vector bosons and fermions

$$
g_{V, f}^{\mathcal{S}}=\xi g_{V, f}^{h_{\mathrm{SM}}}, \quad \text { with } \xi=s_{\alpha}+\frac{v}{f} c_{\alpha} .
$$

\footnotetext{
${ }^{5}$ Note that in the presence of kinetic mixing the transformation from $\left(\Phi, h_{\mathrm{SM}}\right)$ to the mass eigenstates is not orthogonal, and thus $s_{\alpha}$ and $c_{\alpha}$ cannot be expressed as a sine and cosine of a mixing angle (see for instance pp. 56-57 in ref. [37]).
} 
For the dilaton, the couplings $\kappa_{g, \gamma}$ are completely determined using the low-energy theorems and scale invariance [30]. The dilaton coupling to gluons is

$$
\frac{\Phi}{f} \frac{\sum_{\text {heavy }} b_{0}^{i} \alpha_{s}}{8 \pi} G_{\mu \nu} G^{\mu \nu}
$$

where $b_{0}^{i}$ is the contribution of the field $i$ to the QCD $\beta$ function,

$$
\beta_{i}=\frac{b_{0}^{i} g_{s}^{3}}{16 \pi^{2}}
$$

and the sum is over all particles heavier than the scale $f$. Scale invariance implies

$$
\sum_{\text {heavy }} b_{0}^{i}+\sum_{\text {light }} b_{0}^{i}=0 \text {, }
$$

so that we finally obtain

$$
\kappa_{g}=-\sum_{\text {light }} b_{0}^{i}=7
$$

Similarly one obtains [30]

$$
\kappa_{\gamma}=-11 / 3
$$

Note that if we do not include all the SM fields in the conformal sector but keep some of them elementary (e.g., ref. [38]), we cannot use the above arguments to fix $\kappa_{\gamma, g}$ which then become model dependent.

The requirement $f \gtrsim M$ implies

$$
\left|c_{g}\right| \lesssim 21, \quad\left|c_{\gamma}\right| \lesssim 0.7
$$

where we have assumed $s_{\alpha} \sim v / f$ in estimating the small contribution from mixing. For $f \approx M$, the total width, dominated by decays to $W^{+} W^{-}, Z Z, h h$ and $t \bar{t}$, is $\Gamma \approx 30 \mathrm{GeV}$. For this width, eq. (2.8) requires $\left|c_{g} c_{\gamma}\right| \approx 430$ to explain the excess. This cannot be obtained with the numbers in eq. (3.25). We also note that VBF production is negligible, considering the requirement in eq. (2.10).

Thus, we need additional large contributions to the QCD and QED $\beta$ functions below the scale $f$,

$$
\begin{aligned}
\Delta c_{g} & =\frac{2 g_{s}^{2} \Delta b_{\mathrm{QCD}} M}{f}, \\
\Delta c_{\gamma} & =\frac{2 e^{2} \Delta b_{\mathrm{QED}} M}{f} .
\end{aligned}
$$

For $n_{f}$ additional vectorlike colour-triplet, $\mathrm{SU}(2)_{L}$-singlet fermions we have

$$
\begin{aligned}
\Delta b_{\mathrm{QED}} & =\frac{4 N_{c} n_{f}}{3} Q_{f}^{2}, \\
\Delta b_{\mathrm{QCD}} & =\frac{2}{3} n_{f},
\end{aligned}
$$

where $Q_{f}$ is the electric charge of the fermion. Clearly to enhance $c_{g}$ and $c_{\gamma}$ to the extent that $\left|c_{g} c_{\gamma}\right| \approx 430$, we need either a very large charge $Q_{f}$ or a very large number of flavors $n_{f}$ of additional fermions below the TeV scale. This scenario thus appears contrived and we do not investigate it further. 


\subsubsection{Production by quarks}

Finally, we discuss the possibility of production of $\mathcal{S}$ from quarks via the dimension-five operators in eq. (3.1). Thus we consider the Lagrangian terms ${ }^{6}$

$$
\mathcal{L} \supset-\frac{\mathcal{S}}{f}\left(Y_{i j}^{d} H \overline{Q_{i}} d_{j}+Y_{i j}^{u} \tilde{H} \overline{Q_{i}} u_{j}+\text { h.c. }\right)+\kappa_{Y} \frac{\alpha_{1}}{8 \pi} \frac{\mathcal{S}}{f} B_{\mu \nu} B^{\mu \nu} .
$$

We want to find a conservative bound on the maximal energy scale up to which the EFT in eq. (3.30) could be consistent while being completely agnostic about the UV theory. We will consider scenarios in which $\mathcal{S}$ couples primarily to a single quark flavor $f$ and set the corresponding $Y_{i j}^{u, d} \equiv Y_{f}$, as well as $\kappa_{Y}$, to their (conservative) perturbativity bounds as follows:

$$
\frac{Y_{f}}{f} \rightarrow \frac{16 \pi^{2} / \sqrt{N_{c}}}{\Lambda}, \quad \kappa_{Y} \frac{\alpha_{1}}{8 \pi f} \rightarrow \frac{4 \pi}{\Lambda},
$$

so that $\Lambda$ can be identified with the maximum scale up to which the theory could be predictive. ${ }^{7}$ The couplings $c_{f}$ and $c_{\gamma}$ in eq. (2.2) can be expressed in terms of $\Lambda$ as follows,

$$
c_{f}=\frac{16 \pi^{2}}{\sqrt{N_{c}}} \frac{v}{\sqrt{2} \Lambda}, \quad c_{\gamma}=256 \pi^{3} \cos ^{2} \theta_{W} \frac{M}{\Lambda} .
$$

One can find an absolute lower bound on $c_{f}$ by requiring that the production cross section of $\mathcal{S}$ is at least $6.9 \mathrm{fb}$ in accordance with eq. (2.1). For an $f \bar{f}$ initial state with $f=\{u, d, s, c, b\}$ this gives the bounds

$$
\begin{aligned}
& \left|c_{u}\right| \gtrsim 0.005 \Rightarrow \Lambda \lesssim 3200 \mathrm{TeV}, \\
& \left|c_{d}\right| \gtrsim 0.007 \Rightarrow \Lambda \lesssim 2300 \mathrm{TeV}, \\
& \left|c_{s}\right| \gtrsim 0.022 \Rightarrow \Lambda \lesssim 720 \mathrm{TeV}, \\
& \left|c_{c}\right| \gtrsim 0.026 \Rightarrow \Lambda \lesssim 610 \mathrm{TeV}, \\
& \left|c_{b}\right| \gtrsim 0.040 \Rightarrow \Lambda \lesssim 400 \mathrm{TeV},
\end{aligned}
$$

respectively. From eq. (2.10), the coupling to photons, for an $f \bar{f}$ initial state, needs to satisfy

$$
\begin{aligned}
& \left|c_{\gamma}\right| \gtrsim 4.1 \Rightarrow \Lambda \lesssim 1100 \mathrm{TeV}, \\
& \left|c_{\gamma}\right| \gtrsim 5.2 \Rightarrow \Lambda \lesssim 880 \mathrm{TeV}, \\
& \left|c_{\gamma}\right| \gtrsim 17 \Rightarrow \Lambda \lesssim 270 \mathrm{TeV}, \\
& \left|c_{\gamma}\right| \gtrsim 20 \Rightarrow \Lambda \lesssim 230 \mathrm{TeV}, \\
& \left|c_{\gamma}\right| \gtrsim 30 \Rightarrow \Lambda \lesssim 150 \mathrm{TeV},
\end{aligned}
$$

\footnotetext{
${ }^{6}$ Generating the coupling to photons via the $W_{\mu \nu}^{a} W^{a \mu \nu}$ operator instead of $B_{\mu \nu} B^{\mu \nu}$ would require a lower cut-off.

${ }^{7}$ We can think of the following crude picture of how such a large diphoton coupling could be realised. Let us add a vector-like fermion $Q$ as considered in section 3.1.2, but with mass $m_{Q} \sim \Lambda$, charge $Q_{f} \sim 4 \pi / g_{1}$ and Yukawa coupling to $\mathcal{S}$ of $y_{Q} \sim 4 \pi$. This would generate the diphoton coupling in (3.1) with $f \rightarrow \Lambda$ and $\kappa_{Y}$ of order $(4 \pi)^{2} / \alpha_{1}$, which indeed corresponds to the $4 \pi$ in (3.31). This particular scenario does not require any exotic states below $\Lambda$, however generates a very large step in the hypercharge $\beta$ function, which leads to a UV Landau pole for $g_{1}$, and hence for the entire Standard Model, at or close to the scale $\Lambda$.
} 
for $f=\{u, d, s, c, b\}$ respectively, thus giving somewhat stronger bounds than eq. (3.33). The lower bound on $\left|c_{\gamma}\right|$ above can be saturated only in the narrow width case with the additional requirement that $c_{f}$ is a few times higher than the corresponding bound in eq. (3.33) so that the width is dominated by the decays to the production mode (see the discussion below eq. (2.10)). This would require that $\Lambda$ is a few times lower than the bound in eq. (3.33) which roughly coincides with the values in eq. (3.34).

If we assume a $45 \mathrm{GeV}$ width for the resonance, eq. (2.8) must be satisfied, i.e. we must have

$$
\begin{aligned}
&\left|c_{\gamma} c_{u}\right| \approx 2.9 \Rightarrow \Lambda \approx 160 \mathrm{TeV}, \\
&\left|c_{\gamma} c_{d}\right| \approx 3.7 \Rightarrow \Lambda \approx 140 \mathrm{TeV}, \Rightarrow \Lambda \approx 80 \mathrm{TeV}, \\
&\left|c_{\gamma} c_{s}\right| \approx 12 \Rightarrow \Lambda \approx 70 \mathrm{TeV} \\
&\left|c_{\gamma} c_{c}\right| \approx 15 \Rightarrow \Lambda \approx 60 \mathrm{TeV},
\end{aligned}
$$

for an $f \bar{f}$ initial state with $f=\{u, d, s, c, b\}$, respectively, where to obtain the values for the cut-off we have used eq. (3.32).

Note that, in the quark mass basis, the off-diagonal elements of the $Y$ matrices generate terms like $c_{i j} \mathcal{S} \bar{f}_{i} f_{j}$ with $i \neq j$. Tree level FCNC constraints (see, e.g., ref. [39]) constrain these off-diagonal $c_{i j}$ to be $\lesssim \mathcal{O}\left(10^{-4}\right)$ for couplings involving the first two generations and $\lesssim \mathcal{O}\left(10^{-3}\right)$ for couplings involving the $b$ quark, thus much smaller than the values of the diagonal couplings in eq. (3.33). This scenario would thus be interesting from a flavor model-building point of view as one must find a way to suppress the off-diagonal couplings with respect to the diagonal ones. For instance, notice that if $\mathcal{S}$ is a complex scalar, the coupling $c_{i j} \mathcal{S} \bar{f}_{i} f_{j}$ has an accidental flavour symmetry that forbids additional flavor violation, i.e. $\mathcal{S}$ can be formally viewed as a flavon field that carries an $i$ - $j$ flavor charge and thus cannot mediate $\Delta F=2$ flavor violation. In such a case, any flavor violation induced by this coupling is proportional to powers of the $c_{\gamma}$ coupling and/or the SM Yukawas that do not respect this accidental symmetry. The $\Delta F=2$ flavor violation induced by the coupling $c_{i j}$ would thus be suppressed by loop factors and/or SM Yukawas.

Let us now assume that a mechanism for alignment exists thus eliminating any tree level FCNC. In this case flavor violation can arise only at higher loop order. If the production is dominated either by up or down-type $\mathcal{S}$ couplings we can assume that only one of $Y^{u}$ or $Y^{d}$ is non-zero and that it is aligned to the quark mass basis. For instance let us consider the case where in the down mass basis, the production is dominated by a single coupling of $\mathcal{S}$, e.g. $Y_{d}=\operatorname{diag}\left(y_{d}, 0,0\right)$. In such a case flavor violation has to involve the CKM matrix, $V_{\mathrm{CKM}}$. Spurionically, the flavor violating bilinear coupling between two quark doublets is given by $V_{\mathrm{CKM}}^{\dagger} Y_{d}^{\dagger} Y_{d} V_{\mathrm{CKM}}$. This spurion needs to be squared in order to generate the most dangerous $\Delta F=2$ contributions, in this case to $D-\bar{D}$ mixing processes. As $Y_{d}$ is the coefficient of a dimension-five operator in the unbroken phase, each coupling is accompanied with an $\mathcal{S}$ field and thus the term $Y_{d}^{\dagger} Y_{d}$ is generated only at one loop by integrating out $\mathcal{S}$. This holds similarly for the CKM insertions, which can only arise from internal $W$ lines. Consequently, the leading contribution to $\Delta F=2$ (involving quark 
doublets) would be suppressed by a three-loop factor and is thus negligibly small. There are possible two-loop contributions (mixing doublet and singlet quarks) that are, however, suppressed by the light-quark masses and are thus even smaller. Finally, an even stronger (and phenomenologically not necessary) protection is obtained by assuming alignment and $\mathrm{U}(2)$ universality in the form of $Y_{d}=\operatorname{diag}\left(y_{d}, y_{d}, 0\right)$ or $Y_{u}=\operatorname{diag}\left(y_{u}, y_{u}, 0\right)$. In such a case the contributions arise solely via the mixing with the third generation.

\subsection{Excluding the general pure 2HDM}

In this part we discuss the possibility of explaining the excess within the framework of the general two-Higgs-doublet model (2HDM), assuming no additional states beyond the additional doublet.

It is useful to describe the theory in the so-called Higgs basis [40], where only one of the two doublets, which corresponds to the SM Higgs, acquires a VEV. The SM-like Higgs doublet, $H_{a}$, has a VEV equal to $246 \mathrm{GeV}$ and a CP-even component with exactly SM-like couplings, whereas the other doublet, $H_{b}$, which contains the heavy CP-even and CP-odd states, as well as the charged states, has a vanishing VEV. The coupling

$$
-\lambda_{V}\left(H_{b}^{\dagger} H_{a}\right)\left(H_{a}^{\dagger} H_{a}\right)+\text { h.c. }
$$

causes a misalignment between the Higgs basis and the CP-even mass basis [41] that is of order $\lambda_{V} v^{2} / M^{2}$. If $\lambda_{V} \lesssim \mathcal{O}(1)$ we are in the so-called decoupling limit and can think of the ratio $\epsilon \equiv \lambda_{V} v^{2} / M^{2}$ as our formal expansion parameter (see ref. [40] and references therein for relevant discussions). The above interaction term leads to couplings of the heavy CPeven scalar, $H^{0}$, and the pseudoscalar, $A^{0}$, to the electroweak gauge bosons, $V V$. At the same time, it causes deviations from SM values in the $h^{0} V V$ couplings, $h^{0}$ being the lighter CP-even state. The value of $\lambda_{V}$ is thus constrained by electroweak precision measurements. Using the expressions in ref. [42] we find the constraint

$$
\left|\lambda_{V}\right| \lesssim 3
$$

which shows that we are in fact in the decoupling limit as $\epsilon \lesssim 0.3$.

One interesting consequence of the fact that $v^{2} / M^{2} \ll 1$, is that the mass splitting between the neutral CP-even state, $H^{0}$, and the odd one, $A^{0}$, which is due to the coupling

$$
-\frac{\lambda_{5}}{2}\left(H_{a}^{\dagger} H_{b}\right)^{2}+\text { h.c. },
$$

is generically small,

$$
\delta m=\left|m_{H^{0}}-m_{A^{0}}\right| \sim \frac{\left|\lambda_{5}\right| v^{2}}{2 M} \sim 40 \mathrm{GeV}
$$

for $\lambda_{5}=1$. As $\delta m$ is compatible with the width of the excess, one may contemplate the possibility that the observed signal actually arises due to the presence of these two neighbouring states.

We will now show that the general pure $2 \mathrm{HDM}$ cannot account for the observed excess. We note that in the Higgs basis the couplings of the heavy states to the light quarks can 
differ from those of the SM Higgs, as was exploited in ref. [43]; this is because $H_{b}$ acquires no VEV and thus its couplings to the SM fermions do not contribute to their masses. In particular, the couplings of $H_{b}$ to the light quarks might be as large as allowed by the modelindependent constraints in figure 2. Thus, we consider production through either quarkantiquark or gluon fusion. In addition, as the signal might be accounted for by the presence of either $H^{0}$ or $A^{0}$, or both, we should consider the production and decay of each of these. We emphasise that to be conservative we do not require the width to be equal to $45 \mathrm{GeV}$ as the excess could be explained by two narrower states separated in mass by a few tens of GeVs, which would be consistent with the reported diphoton spectrum. We denote by $N_{H}$ and $N_{A}$ the number of events from the production and decay of $H^{0}$ and $A^{0}$, respectively. In the CP limit we can assume no interference between these two production modes.

Gluon-gluon production. Assuming that the masses of $A^{0}$ and $H^{0}$ are less than $45 \mathrm{GeV}$ apart, both states would contribute to the excess. For the total width of the resonance to not exceed $45 \mathrm{GeV}$, eq. (2.3), it is necessary that

$$
Y^{2} \equiv \sum_{f} \beta_{f} c_{f}^{2} \lesssim 0.5
$$

where $c_{f}$ is the coupling of $H^{0}$ and $A^{0}$ to the SM fermions,

$$
-c_{f} \bar{f}_{L}\left(H^{0}+i A^{0}\right) f_{R}+h . c .
$$

and $\beta_{f}=\left(1-4 m_{f}^{2} / M^{2}\right)^{1 / 2}$, with $m_{f}$ being the fermion mass. Taking into account the steep decrease of the fermion loop functions $\tilde{A}_{1 / 2}$ and $\tilde{A}_{1 / 2}^{P S}$, defined respectively in eqs. (3.7) and (3.16), with decreasing quark mass, we find that for a fixed partial width to fermions (and thus fixed $Y^{2}$ ), the fermionic loop contributions to $c_{\gamma}$ and $c_{g}$ are maximized for $c_{t} / c_{f^{\prime}} \gg 1$, where $c_{t}$ is the coupling to the top and $c_{f^{\prime}}$ are couplings to fermions other than the top.

It is possible to bound the contributions from $A^{0}$ because, unlike for $H^{0}$, its couplings to the photons and gluons are only due to fermion loops. The total number of events from pseudoscalar decays can be expressed using eq. (2.4) as

$$
N_{A}=8.0 \times 10^{5} \times \mathrm{BR}\left(A^{0} \rightarrow g g\right) \times \mathrm{BR}\left(A^{0} \rightarrow \gamma \gamma\right) \times \frac{\Gamma\left(A^{0}\right)}{45 \mathrm{GeV}} .
$$

Using the inequalities

$$
\mathrm{BR}\left(A^{0} \rightarrow g g\right)<\frac{\Gamma\left(A^{0} \rightarrow g g\right)}{\Gamma\left(A^{0} \rightarrow f \bar{f}\right)}, \quad \operatorname{BR}\left(A^{0} \rightarrow \gamma \gamma\right)<\frac{\Gamma\left(A^{0} \rightarrow \gamma \gamma\right)}{\Gamma\left(A^{0} \rightarrow f \bar{f}\right)}
$$

along with the condition $\Gamma\left(A^{0}\right) \lesssim 45 \mathrm{GeV}$ we then obtain

$$
N_{A} \lesssim 8.0 \times 10^{5} \times \frac{\Gamma\left(A^{0} \rightarrow g g\right) \times \Gamma\left(A^{0} \rightarrow \gamma \gamma\right)}{\Gamma\left(A^{0} \rightarrow f \bar{f}\right)^{2}}
$$


The partial widths are given by

$$
\begin{aligned}
& \Gamma\left(A^{0} \rightarrow g g\right)=\frac{\alpha_{s}^{2} M}{32 \pi^{3}}\left|\sum_{f} c_{f} \tilde{A}_{1 / 2}^{P S}\left(\tau_{f}\right)\right|^{2}, \\
& \Gamma\left(A^{0} \rightarrow \gamma \gamma\right)=\frac{\alpha^{2} M}{64 \pi^{3}}\left|\sum_{f} c_{f} N_{c} Q_{f}^{2} \tilde{A}_{1 / 2}^{P S}\left(\tau_{f}\right)\right|^{2},
\end{aligned}
$$

where $\tau_{f}=M^{2} /\left(4 m_{f}^{2}\right)$ and $\tilde{A}_{1 / 2}^{P S}$ is defined in eq. (3.16). Taking $c_{t} \gg c_{f^{\prime}}$, as explained above, one can now evaluate the upper bound in eq. (3.44),

$$
N_{A} \lesssim 0.02
$$

where we have used $\Gamma\left(A^{0} \rightarrow t \bar{t}\right)=\frac{3}{8 \pi} \sqrt{1-4 m_{t}^{2} / M^{2}} M c_{t}^{2} \approx 0.11 M c_{t}^{2}$. We thus conclude that the pseudoscalar contributions are negligibly small in this case.

We must then attribute all 20 signal events to $H^{0}$ decays,

$$
20=N_{H}<1.8 \times 10^{4} \mathrm{GeV}^{-1} \times \frac{\Gamma\left(H^{0} \rightarrow g g\right)}{\Gamma\left(H^{0} \rightarrow f \bar{f}\right)} \times \Gamma\left(H^{0} \rightarrow \gamma \gamma\right),
$$

where we have used eq. (2.4) and $\Gamma\left(H^{0} \rightarrow f \bar{f}\right)<\Gamma\left(H^{0}\right)$. Now, as above, we take $c_{t} \gg c_{f^{\prime}}$ in

$$
\Gamma\left(H^{0} \rightarrow g g\right)=\frac{\alpha_{s}^{2} M}{128 \pi^{3}}\left|\sum_{f} c_{f} \tilde{A}_{1 / 2}\left(\tau_{f}\right)\right|^{2}
$$

to maximise the ratio $\Gamma\left(H^{0} \rightarrow g g\right) / \Gamma\left(H^{0} \rightarrow f \bar{f}\right)$, which becomes independent of $c_{f}$. Using $\Gamma\left(H^{0} \rightarrow \gamma \gamma\right)=1.99 \times 10^{-7} M\left|c_{\gamma}\right|^{2}$ from table 1 we then obtain the requirement

$$
\left|c_{\gamma}\right| \gtrsim 66
$$

As we will soon show, such large values of $\left|c_{\gamma}\right|$ are impossible to obtain in a pure 2HDM.

Quark-antiquark production. As argued above, in general the heavy states can have sizeable couplings to the first two generations. Ignoring possible severe constraints from flavor physics we find that the weakest bound is from production due to $u \bar{u}$. Again we bound the pseudoscalar contribution first. Using eq. (2.4) we have

$$
N_{A}=8.1 \times 10^{3} \mathrm{GeV}^{-1} \times \frac{\Gamma\left(A^{0} \rightarrow u \bar{u}\right) \Gamma\left(A^{0} \rightarrow \gamma \gamma\right)}{\Gamma\left(A^{0}\right)} .
$$

As the up-quark loop contributes negligibly to $\Gamma\left(A^{0} \rightarrow \gamma \gamma\right)$ compared with the top-quark loop, the above expression is proportional to $c_{u}^{2} c_{t}^{2} / Y^{2}$ assuming that all the other fermionic couplings are zero. Keeping the bound from eq. (3.40) in mind, this is maximised for $c_{u}^{2}=0.25$ and $c_{t}^{2}=0.28$. For these values the pseudoscalar contribution yields less than one event. Thus $H^{0}$ must account for all 20 events. From eq. (2.10) we have the requirement

$$
\left|c_{\gamma}\right| \gtrsim 4.1 \text {. }
$$


Let us now discuss whether $\left|c_{\gamma}\right|$ as large as that required by eqs. (3.50) and (3.52) can be obtained by loops of charged particles for the $H^{0}$ in the pure 2HDM. In addition to fermionic loops, the couplings of $H^{0}$ to photons receive contributions from loops of $W^{ \pm}$ and $H^{ \pm}$. In the Higgs basis the two couplings that can parametrize these contributions are $\lambda_{V}$, defined in eq. (3.37), and

$$
-\lambda_{H^{+}}\left(H_{a}^{\dagger} H_{b}\right)\left(H_{b}^{\dagger} H_{b}\right)+\text { h.c. },
$$

where the term proportional to $\lambda_{H^{+}}\left(\lambda_{V}\right)$ results in a coupling of the $H^{0}$ to the charged Higgs $(W)$. We take the maximal value of $\lambda_{V}$ allowed by electroweak precision constraints, $\lambda_{V} \approx 3$, as already mentioned above. There is no analogous restriction on the value of $\lambda_{H^{+}}$. To check whether it is possible to satisfy the requirement in eq. (3.50), or at least the one in eq. (3.52), we have added up the loop contributions from the top quark, the $W$ and the charged Higgs (see, e.g., ref. [32]) allowing for maximal constructive interference. To maximise $\left|c_{\gamma}\right|$, we take the charged-Higgs mass to be as small as $M / 2$, which can, for instance, be obtained with a large value of $\lambda_{5}$. For $\mathcal{O}(1)$ values of $\lambda_{H^{+}}$, the contribution of the charged-Higgs loop is very small compared to the dominant contribution from the top loop as it is suppressed by $m_{W}^{2} / m_{H^{+}}^{2}$. We get, for $\lambda_{H^{+}}=1,\left|c_{\gamma}\right| \sim 1.8$. We find that to satisfy even the bound $\left|c_{\gamma}\right| \gtrsim 4.1$ in eq. (3.52) requires very large values of $\lambda_{H^{+}}$, above $16 \pi^{2} / 3$. For such large values of $\lambda_{H^{+}}$, a naive estimate tells us that the loop contributions are a third of the tree-level ones, so perturbativity is questionable. Such large values of $\lambda_{H^{+}}$and $\lambda_{5}$ are also ruled out if we require their contribution to the running of $\lambda_{V}$ between the scales $M$ and $m_{Z}$ to be smaller than the electroweak precision bound (which applies to $\lambda_{V}\left(m_{Z}\right)$ ), that is if we require $\Delta \lambda_{V} \lesssim 3$ (see ref. [42] for the RGE). This rules out both gluon and quark initiated production as the bounds in eqs. (3.50) and (3.52) are impossible to satisfy.

Thus, we have verified that the general $2 \mathrm{HDM}$, without any additional states, cannot account for the observed anomaly.

\subsection{The fate of the MSSM}

We now turn to the Minimal Supersymmetric Standard Model (MSSM). As in the 2HDM, which in its type-II form is contained in the MSSM as a subsector, the only candidate particles for the resonance in the MSSM are $H^{0}$ and $A^{0} .^{8}$ The most plausible production mechanism is gluon fusion, due to the smallness of the $H_{d}$ doublet's Yukawa couplings to light quarks and the fact that we are deep in the decoupling regime, $M_{H^{0}} \gg m_{Z}$.

As we have seen above, the $2 \mathrm{HDM}$ fails by a large margin to accommodate the data. However, in the MSSM there are extra contributions to the $H^{0} g g$ couplings from sfermions

\footnotetext{
${ }^{8}$ We consider the R-parity-conserving MSSM, otherwise in principle one could consider sneutrino candidates, which can be similarly constrained. A resonant $\gamma \gamma$ signal can also arise within the MSSM from the annihilation of a squark-antisquark near-threshold QCD bound state, most famously the stoponium [44]. However, based on expressions from [45], the stoponium has $\left|c_{\gamma}\right| \simeq \sqrt{\left(2^{21} \pi^{5} / 3^{6}\right) \bar{\alpha}_{s}^{3} \alpha^{2}} \approx 0.4$, while eq. (2.10) requires $\left|c_{\gamma}\right| \gtrsim 2.7$ even for the most favorable (but also a quite generic for stoponium) scenario where the width is dominated by decays to the production mode, $\Gamma_{g g} \simeq(16 / 81) \bar{\alpha}_{s}^{3} \alpha_{s}^{2} M \approx 0.0033 \mathrm{GeV}$. One might also consider the gluinonium, whose binding is much stronger, though annihilation to $\gamma \gamma$ is loop-suppressed [45, 46]. However, pair production of $M / 2 \approx 375 \mathrm{GeV}$ gluinos would have been almost certainly noticed by now.
} 
and to the $H^{0} \gamma \gamma$ couplings from sfermions and charginos, in addition to those already present in the $2 \mathrm{HDM}$. The $A^{0} g g$ and $A^{0} \gamma \gamma$ vertices receive no sfermion contributions at one loop as a consequence of $\mathrm{CP}$ symmetry, though they do receive contributions from charginos. ${ }^{9}$ Considering first $H^{0}$ as a candidate, dimensional analysis gives, for the contribution of the two stops, for $M_{\mathrm{SUSY}}=1 \mathrm{TeV}$,

$$
c_{g} \sim 2 g_{s}^{2} \times \frac{v M_{H^{0}}}{M_{\mathrm{SUSY}}^{2}} \sim 0.5
$$

and

$$
c_{\gamma} \sim 2 N_{c} e^{2} \times \frac{v M_{H^{0}}}{M_{\text {SUSY }}^{2}} \sim 0.1
$$

Even allowing for similar contributions from other sparticles, this suggests that, generically, $\left|c_{g} c_{\gamma}\right|<1$, which is nearly three orders of magnitude below what is required according to eq. (2.8). However, we must also contemplate that the true resonance width could be smaller than the "nominal" $45 \mathrm{GeV}$. The decay width of $H^{0}$ is dominated by tree-level decays into top and bottom quarks, and is essentially determined in the MSSM as a function of $\tan \beta$, with a minimum of about $2 \mathrm{GeV}$ at $\tan \beta \approx 6$. Hence, eq. (2.8) can be recast as

$$
\frac{\left|c_{\gamma} c_{g}\right|}{\sqrt{\Gamma(\tan \beta) /(45 \mathrm{GeV})}}=\rho_{g} \approx 530
$$

The question is how large the left-hand side may be. First, a small numerator could be partly compensated for by a factor of up to five due to the denominator. Second, an MSSM spectrum could also be quite non-degenerate, with hierarchies like $m_{\tilde{t}_{1}} \ll M_{H^{0}}, \mu \ll m_{\tilde{t}_{2}}$; this is in fact favoured by the observed Higgs mass. In particular, large $\mu$ and/or $A$-terms and a light stop can lead to a parametric enhancement $\sim\left\{\mu, A_{t}\right\} / m_{\tilde{t}_{1}}$ relative to the naive estimates above. Third, there could also be important contributions from sbottoms and staus, as well as charginos, which brings in a large subset of the MSSM parameters. A conclusion about the fate of the MSSM requires a quantitative treatment, but a brute-force parameter scan is not really feasible and in any case beyond the scope of this work. Instead, the purpose of the rest of this section is to obtain simple yet conservative bounds on all one-loop contributions over the entire MSSM parameter space.

First, we will impose $1 \leq \tan \beta \leq 50$. The reason is that in the decoupling limit the $H^{0} t \bar{t}$ and $H^{0} b \bar{b}$ couplings are $\sqrt{2} m_{t} /(v \tan \beta)$ and $\sqrt{2} m_{b} /(v \cot \beta)$, respectively, ${ }^{10}$ which, outside the stated $\tan \beta$ range, implies a decay width that significantly exceeds the width allowed by observations, cf. section 2.1. (Independently, such large couplings would lead to a Landau pole in $y_{t}$ or $y_{b}$, and/or strong coupling at low scales. Our lower limit on $\tan \beta$ also has very strong support from the observed Higgs mass of $125 \mathrm{GeV}$, which we will not separately impose.) The key assumption will be the absence of charge- and colour-breaking

\footnotetext{
${ }^{9}$ As in the rest of this work, we assume $C P$ conservation. Without this assumption, the gluonic and photonic couplings of some superposition of the two heavier mass eigenstates $H_{2}$ and $H_{3}$ will receive sparticle loop contributions, so apart from a division of the diphoton signal between $H_{2}$ and $H_{3}$ resonant contributions, we do not expect qualitative changes to our conclusions.

${ }^{10}$ For this subsection, we use a convention $v \approx 174 \mathrm{GeV}$.
} 
minima of the scalar potential. This could in principle be relaxed to only require metastability over cosmological timescales; we leave this aside for future work. As we will see, this assumption is sufficient to exclude the MSSM if the resonance interpretation is confirmed.

\subsubsection{Constraints from vacuum stability}

An essential role in our argument is played by the upper bounds on the $\mu$ parameter and the soft trilinear terms that follow from requiring the absence of charge- and colour-breaking minima of the MSSM scalar potential. The derivation of these bounds is well known [47-54] and involves suitable directions of the MSSM scalar field space. We employ five such directions

$$
T_{L}=T_{R}=H_{u}^{0}, \quad B_{L}=B_{R}=H_{d}^{0}, \quad T_{L}=T_{R}=H_{d}^{0}, \quad B_{L}=B_{R}=H_{u}^{0}, \quad \mathcal{T}_{L}=\mathcal{T}_{R}=H_{u}^{0},
$$

(with all other scalar fields held at zero), of which the first two are $D$-flat. The five bounds derived from these directions can be formulated in terms of the stop, sbottom and stau masses, as:

$$
\begin{aligned}
& \left|A_{t}\right| \leq \sqrt{3} \sqrt{m_{\tilde{t}_{1}}^{2}+m_{\tilde{t}_{2}}^{2}-2 m_{t}^{2}+\frac{M_{H^{0}}^{2}}{2}\left(1+c_{2 \beta}\right)-\frac{m_{Z}^{2}}{2}\left(1-c_{2 \beta}\right)\left(1+c_{2 \beta}\right)^{2}} \\
& \left|A_{b}\right| \leq \sqrt{3} \sqrt{m_{\tilde{b}_{1}}^{2}+m_{\tilde{b}_{2}}^{2}-2 m_{b}^{2}+\frac{M_{H^{0}}^{2}}{2}\left(1-c_{2 \beta}\right)-\frac{m_{Z}^{2}}{2}\left(1-c_{2 \beta}\right)^{2}\left(1+c_{2 \beta}\right)}, \\
& |\mu| \leq \sqrt{1+\frac{m_{Z}^{2}}{m_{t}^{2}} \sin ^{2} \beta} \\
& \times \sqrt{m_{\tilde{t}_{1}}^{2}+m_{\tilde{t}_{2}}^{2}-2 m_{t}^{2}+\frac{M_{H^{0}}^{2}}{2}\left(1-c_{2 \beta}\right)-\frac{m_{Z}^{2}}{2}\left(1+c_{2 \beta}-c_{2 \beta}^{2}+c_{2 \beta}^{3}\right)}, \\
& m_{b}|\mu| \tan \beta \leq m_{t} \sqrt{\frac{\tan ^{2} \beta}{R^{2}}+\frac{m_{Z}^{2}}{m_{t}^{2}} \sin ^{2} \beta} \\
& \times \sqrt{m_{\tilde{b}_{1}}^{2}+m_{\tilde{b}_{2}}^{2}-2 m_{b}^{2}+\frac{M_{H^{0}}^{2}}{2}\left(1+c_{2 \beta}\right)-\frac{m_{Z}^{2}}{2}\left(1-c_{2 \beta}-c_{2 \beta}^{2}-c_{2 \beta}^{3}\right)}, \\
& m_{\tau}|\mu| \tan \beta \leq m_{t} \sqrt{\frac{\tan ^{2} \beta}{R_{\tau}^{2}}+\frac{m_{Z}^{2}}{m_{t}^{2}} \sin ^{2} \beta} \\
& \times \sqrt{m_{\tilde{\tau}_{1}}^{2}+m_{\tilde{\tau}_{2}}^{2}-2 m_{\tau}^{2}+\frac{M_{H^{0}}^{2}}{2}\left(1+c_{2 \beta}\right)-\frac{m_{Z}^{2}}{2}\left(1-c_{2 \beta}-c_{2 \beta}^{2}-c_{2 \beta}^{3}\right)},
\end{aligned}
$$

where $R \equiv m_{t} / m_{b} \sim 50, R_{\tau} \equiv m_{t} / m_{\tau} \sim 100$, and $c_{2 \beta} \equiv \cos (2 \beta)$. Eq. (3.59) also has an analogue for $A_{\tau}$, obtained by substituting $b \rightarrow \tau$.

In these expressions we have kept the exact dependence on $\beta$, but neglected small terms of order $m_{Z}^{4} / M_{H^{0}}^{4}$ (i.e. we have taken the decoupling limit). Also, we have employed tree-level mass relations; this can easily be undone (for example, $m_{b}|\mu| \tan \beta \rightarrow y_{b}|\mu|$ on the left-hand side of eq. (3.61), and $m_{t} \sqrt{\tan ^{2} \beta / R^{2}+m_{Z}^{2} / m_{t}^{2} \sin ^{2} \beta} \rightarrow y_{t} \sqrt{y_{b}^{2}+\left(g^{2}+g^{\prime 2}\right) / 2}$ on its right-hand side). 
We can combine the bounds into bounding functions $\Phi_{t}, \Phi_{b}$, and $\Phi_{\tau}$ of the sfermion masses and $\beta$ only. Firstly,

$$
\Phi_{t}=\left\{\begin{array}{c}
0, \quad m_{\tilde{t}_{1}}^{2}+m_{\tilde{t}_{2}}^{2}-2 m_{t}^{2}+\frac{M_{H^{0}}^{2}}{2}\left(1+c_{2 \beta}\right)-\frac{m_{Z}^{2}}{2}\left(1-c_{2 \beta}\right)\left(1+c_{2 \beta}\right)^{2}<0, \\
\sqrt{3} \sqrt{m_{\tilde{t}_{1}}^{2}+m_{\tilde{t}_{2}}^{2}-2 m_{t}^{2}+\frac{M_{H^{0}}^{2}}{2}\left(1-c_{2 \beta}\right)-\frac{m_{Z}^{2}}{2}\left(1+c_{2 \beta}-c_{2 \beta}^{2}+c_{2 \beta}^{3}\right)}, \quad \text { otherwise. }
\end{array}\right.
$$

If the condition for $\Phi_{t}=0$ is satisfied, there is no way to satisfy the $A_{t}$ constraint; setting the bounding function to zero in this case will serve to effectively discard those unphysical points below. Otherwise, $\Phi_{t}$ simultaneously bounds both $\left|A_{t}\right|$ and $|\mu|$. A similar function that simultaneously bounds $\left|A_{b}\right|$ and $\frac{m_{b}}{m_{t}}|\mu| \tan \beta$ is provided by

$$
\Phi_{b}=\sqrt{3} \sqrt{m_{\tilde{b}_{1}}^{2}+m_{\tilde{b}_{2}}^{2}-2 m_{b}^{2}+\frac{M_{H^{0}}^{2}}{2}\left(1-c_{2 \beta}\right)},
$$

and an identical function $\Phi_{\tau}$ follows from this by substituting $b \rightarrow \tau$.

\subsubsection{Conservative bounds on sfermion contributions}

In the notation of ref. [55], the sfermion contributions to $c_{g}$ are given by

$$
\left|\sum_{f} c_{g}^{(\tilde{f})}\right|=\frac{g M_{H^{0}}}{2 M_{W}} g_{s}^{2}\left|A_{\mathrm{SUSY}, \tilde{f}}^{H^{0}}\right| .
$$

If the contribution of a sfermion to $c_{g}$ is known, the corresponding contribution to $c_{\gamma}$ is given by

$$
c_{\gamma}^{(\tilde{f})}=2\left(e^{2} / g_{s}^{2}\right) N_{c}^{(f)} Q_{f}^{2} c_{g}^{(\tilde{f})} .
$$

Explicitly,

$$
A_{\mathrm{SUSY}, \tilde{f}}^{H^{0}}=4 \sum_{\tilde{f}=\tilde{t}, \tilde{b}, \tilde{\tau}} \sum_{i=1,2} \frac{g_{\tilde{f}_{i} \tilde{f}_{i}}^{H^{0}}}{M_{H^{0}}^{2}} h\left(\tau_{i}^{\tilde{f}}\right)
$$

where $\tau_{i}^{\tilde{f}}=M_{H^{0}}^{2} /\left(4 m_{\tilde{f}_{i}}^{2}\right)$ and

$$
h(\tau)=\tau A_{0}^{H^{0}}(\tau)=\left\{\begin{array}{cc}
\frac{\arcsin ^{2}(\sqrt{\tau})}{\tau}-1 & \tau \leq 1 \\
-\frac{1}{4 \tau}\left(\ln \frac{1+\sqrt{1-\frac{1}{\tau}}}{1-\sqrt{1-\frac{1}{\tau}}}-i \pi\right)^{2}-1 & \tau>1 .
\end{array}\right.
$$

Consider first the stops. In the decoupling limit, their couplings to $H^{0}$ are

$$
g_{\tilde{t}_{i} \tilde{t}_{i}}^{H^{0}}=-\cot \beta m_{t}^{2}+x_{i} \sin (2 \beta) m_{Z}^{2} \pm m_{t} \frac{\sin \left(2 \theta_{\tilde{t}}\right)}{2}\left(\mu+A_{t} \cot \beta\right),
$$

where $\theta_{\tilde{t}}$ is the stop mixing angle, and the coefficients $x_{i}$ depend on $\theta_{\tilde{t}}$ and $\beta$ and are always less than one in magnitude. Using that $h \rightarrow 0$ for $\tau \rightarrow 0, \infty$ and $|h| \leq h(1) \approx 1.47$, one easily shows that the first two terms lead to maximal contributions to $A_{\mathrm{SUSY}, \tilde{f}}^{H^{0}}$ that 
are bounded (in magnitude) by $2.74 \cot \beta$ and 0.03 , respectively. (Similar terms for the sbottom and stau cases will be negligible.) The third term in the coupling leads to

$$
A_{\mathrm{SUSY}, \tilde{t}}^{H^{0}}=\frac{\sin \left(2 \theta_{\tilde{t}}\right)}{2} \frac{4 m_{t}\left(A_{t} \cot \beta+\mu\right)}{M_{H^{0}}^{2}} \times\left(h\left(\tau_{1}\right)-h\left(\tau_{2}\right)\right) .
$$

Employing now the bounding function $\Phi_{t}$ from the previous subsection, it is not difficult to show that

$$
\left|m_{t} \frac{\sin \left(2 \theta_{\tilde{t}}\right)}{2}\left(A_{t} \cot \beta+\mu\right)\right| \leq m_{t} \min \left(\frac{1}{2} \Phi_{t}(1+\cot \beta), m_{t} \frac{\Phi_{t}^{2}}{m_{\tilde{t}_{2}}^{2}-m_{\tilde{t}_{1}}^{2}}\right) \equiv \frac{M_{H^{0}}^{2}}{4} B\left(\tau_{1}, \tau_{2} ; M_{H^{0}}\right) .
$$

The first argument of the min function follows from $|\sin | \leq 1$, the second makes use of the explicit formula for the stop mixing angle. We then have

$$
\left|A_{\mathrm{SUSY}, \tilde{t}}^{H^{0}}\right| \leq B\left(\tau_{1}, \tau_{2}\right)\left|h\left(\tau_{1}\right)-h\left(\tau_{2}\right)\right| .
$$

The right-hand side is bounded and the physical parameter space is the compact region $0 \leq \tau_{i} \leq \tau_{i}^{\max }$, where $\tau_{i}^{\max }=M_{H^{0}}^{2} /\left(4 m_{\tilde{t}}^{\min }\right)$ depends on the experimental lower bound on the lighter stop mass. Straightforward numerical techniques establish that

$$
\left|A_{\mathrm{SUSY}, \tilde{t}}^{H^{0}}\right| \leq 3.37
$$

where the maximum is obtained at $\tan \beta=1$, when one stop is at threshold $\left(m=M_{H} / 2\right)$ and the other is relatively light. Below, we numerically obtain and use the bounds as a function of $\tan \beta$. (We allow stop masses as light as $100 \mathrm{GeV}$ in the scan, to escape any doubts related to for instance compressed spectra where light stops might have escaped detection at the LHC). The extremal point is generally ruled out by the observed Higgs mass $m_{h}=125 \mathrm{GeV}$, and very unlikely to be consistent with LHC searches, but we are being conservative.

Analogous steps lead to bounds on the sbottom and stau contributions. In this case, terms proportional to $m_{b, \tau}^{2}$ and $m_{Z}^{2}$ in the Higgs-sfermion couplings lead to completely negligible effects. For the remainder, we require a bound

$$
\begin{aligned}
\left|m_{b} \frac{\sin \left(2 \theta_{\tilde{b}}\right)}{2}\left(\mu-A_{b} \tan \beta\right)\right| \leq & m_{t} \min \left(\frac{1}{2} \Phi_{b}\left[\cot \beta+\frac{\tan \beta}{R}\right]\right. \\
& \left.m_{t} \frac{\Phi_{b}^{2}}{m_{\tilde{b}_{2}}^{2}-m_{\tilde{b}_{1}}^{2}}\left[\cot \beta+\frac{\tan \beta}{R}\right]\left[1+\frac{1}{R}\right]\right) \\
& \equiv \frac{M_{H^{0}}^{2}}{4} B_{\tilde{b}}\left(\tau_{1}, \tau_{2} ; M_{H^{0}}\right) .
\end{aligned}
$$

The resulting bound is most effective in the intermediate $\tan \beta$ region, counteracting the small denominator of eq. (3.56) in that region. The bound on the stau contribution, as a function of $\tan \beta$ and the slepton masses, is identical to the sbottom one, except for a missing colour factor (overcompensated in the photonic coupling by a ninefold larger squared electric charge). 


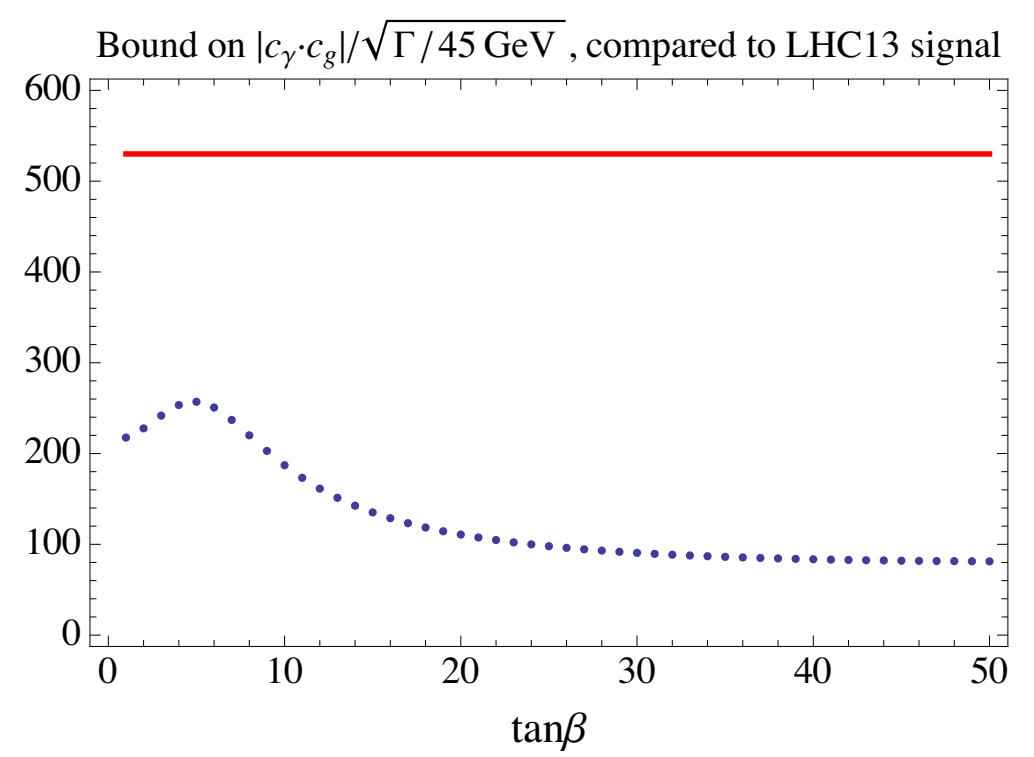

Figure 7. Comparison of the upper bound on the left-hand side of eq. (3.56) to the signal suggested by the diphoton excesses, as a function of $\tan \beta$. The red horizontal line corresponds to the signal, and the blue dots represent our conservative upper bound.

\subsubsection{Contributions from other particles and verdict}

The contributions from top, bottom, $W$, and charged-Higgs loops have already been discussed in the $2 \mathrm{HDM}$ section. In the decoupling limit, where $M_{H^{+}} \approx M_{H^{0}}$, they are essentially functions of $\tan \beta$ only and easily incorporated. Regarding charginos, their effect is equivalent to the contribution of two vectorlike, colourless particles; such contributions have also been discussed above. We only need to bound the fermion loop function by its global maximum and make no use of the relation of the chargino and Higgs mixing angles to MSSM parameters in order to obtain the bound $\left|c_{\gamma}^{\chi^{+}}\right| \leq 0.45$ (for any $\tan \beta$ ). Assuming now the extreme scenario where all contributions to $c_{\gamma}$ and $c_{g}$ simultaneously saturate their bounds and are in phase with one another, we obtain a (very) conservative upper bound on the left-hand side of eq. (3.56). This is displayed in figure 7. We observe that this bound still misses the data by more than a factor of two, even at the point of closest approach at $\tan \beta \sim 5$. It is fairly clear that the bound could be made stronger by, for example, employing more properties of the function $h$ or formulating a higher-dimensional extremization problem (closer to a full scan of the MSSM parameter space). It is also clear that the pseudoscalar $A^{0}$ fares worse than $H^{0}$ as a resonance candidate: the chargino contribution to its coupling to photons is similarly constrained as in the $H^{0}$ case, while sfermion contributions to both the photonic and gluonic couplings are absent, giving a much tighter bound on the left-hand side of eq. (3.56) in this case.

\subsubsection{Production from quarks?}

So far we only considered the production from gluons. A similar leading-order analysis for quark-antiquark initial states again leads to a negative conclusion. The bounds just 
established translate to an upper bound $\left|c_{\gamma}\right|<5.3$, attained (for $\tan \beta \geq 1$ ) at $\tan \beta=$ 1. This can be combined with the model-independent analysis of section 2. First, the constraint in eq. (2.10) rules out initial states other than $u \bar{u}$ or $d \bar{d}$. Eq. (2.18) then implies $(\Gamma /(45 \mathrm{GeV}))^{1 / 4}<0.5$, which together with eq. (2.17) implies $\left|c_{u}\right|<0.15$ for $u \bar{u}$ initial state $\left(\left|c_{d}\right|<0.18\right.$ for $d \bar{d}$ initial state). (The couplings $c_{u}$ and $c_{d}$ denote the Yukawa couplings of the scalar mass eigenstate $H^{0}$, as defined in section 2. For finite $\tan \beta$ this state is a superposition of the neutral components of $H_{u}$ and $H_{d}$, and yet another superposition of the doublets in the "Higgs basis" of the preceding subsections.) At the same time, the signal constraint together with the expression for the width-to-mass ratio (eqs. (2.8) and (2.3), respectively) imply

$$
\left|c_{f}\right|>\frac{2.9(3.7)}{\left|c_{\gamma}\right|} \sqrt{\left(n_{\gamma \gamma}\left|c_{\gamma}\right|^{2}+n_{t}\left|c_{t}\right|^{2}+n_{b}\left|c_{b}\right|^{2}\right) \frac{750 \mathrm{GeV}}{45 \mathrm{GeV}}} .
$$

Using the tree-level relations $\left|c_{t}\right|=\frac{m_{t}}{\sqrt{2} v} \cot \beta,\left|c_{b}\right|=\frac{m_{b}}{\sqrt{2} v} \tan \beta$, we find this to be in conflict with the upper bound unless $3<\tan \beta<15$ for $u \bar{u}$ initial state $(4<\tan \beta<14$ for $d \bar{d}$ initial state), in which case $\left|c_{u}\right|>0.10$ (or $\left|c_{d}\right|>0.13$ ). Employing again the tree-level relations, these $\tan \beta$ ranges correspond to an up-quark mass above $100 \mathrm{GeV}$ (down-quark mass above a few $\mathrm{GeV}$ ), both in gross contradiction with observation.

However, higher-order corrections in the MSSM could potentially affect our conclusions. Although it is hard to see how they could give $\mathcal{O}(1)$ or larger corrections to the $H^{0} g g$ or $H^{0} \gamma \gamma$ vertices, loop corrections can contribute $\mathcal{O}(1)$ fractions of the down-type quark masses, through an induced coupling to the doublet $H_{u} \cdot{ }^{11}$ In this case, $c_{b}$ entering eq. (3.75) is no longer determined by $m_{b}$ and $\tan \beta$, and so for $\tan \beta \rightarrow \infty$ one would have only a very weak bound $\left|c_{u}\right|>0.005\left(\left|c_{d}\right|>0.007\right)$ due to the partial width into diphotons. While a complete investigation goes beyond the methodology and scope of this paper, we can put some relevant restrictions on such a scenario.

The fact that $\tau \tau$ resonance searches do not show an excess results in an upper bound on the tree-level $\tau$ mass, giving

$$
m_{\tau}^{\text {tree }}<\frac{7.4}{\tan \beta} \mathrm{GeV}
$$

This follows directly from the upper bound on the ratio $\mathrm{BR}_{\tau \tau} / \mathrm{BR}_{\gamma \gamma}=\left(n_{\tau} / n_{\gamma}\right)\left(\left|c_{\tau}\right|^{2} /\left|c_{\gamma}\right|^{2}\right)$ (cf. eq. (2.16) and table 3), using $\left|c_{\gamma}\right|>5.3$, giving $\left|c_{\tau}\right|<0.026$. (This might be relaxed to about 0.03 for a mix of $g g$ and $q \bar{q}$ production.) This implies that either $\tan \beta<10$ or the dominant fraction of the $\tau$ mass would have to come from one-loop contributions.

Such one-loop contributions have been considered in the literature (see, e.g., ref. [56]) and are due to neutralino-stau and chargino-sneutrino loops, with the latter suppressed by the small $\left|y_{\tau}\right|=\sqrt{2}\left|c_{\tau}\right| / \sin \beta<0.06$. Discarding them, the remaining neutralino-stau contributions are proportional to the left-hand side of eq. (3.62) times a combination of coupling constants, times a loop function. ${ }^{12}$ For $\tan \beta>8.3$, the $M_{H^{0}}$ and $m_{Z}$ dependence of the stability bound of eq. (3.62) can be conservatively dropped. The one-loop

\footnotetext{
${ }^{11}$ We thank Martin Gorbahn for stressing this to us.

${ }^{12}$ If nonholomorphic soft terms are allowed, the left-hand side of eq. (3.62) is modified but remains proportional to the relevant $\tilde{\tau} \tilde{\tau} H_{u}^{0}$ coupling, such that the coupling remains bounded by the right-hand side.
} 
contribution of a given neutralino to the $\tau$ mass is then bounded by the dimensionless combination

$$
\sqrt{m_{\tilde{\tau}_{1}}^{2}+m_{\tilde{\tau}_{2}}^{2}} m_{\chi_{0}} I\left(m_{\tilde{\tau}_{1}}^{2}, m_{\tilde{\tau}_{2}}^{2}, m_{\chi_{0}}^{2}\right)
$$

(with $I$ defined in [56]), which is globally bounded in magnitude by one, times a factor independent of sparticle masses. Summing the latter over neutralinos and maximizing over mixing angles, we find that $\Delta m_{\tau}^{1 \text {-loop }}<0.2 \mathrm{GeV}$ for $\tan \beta>8.3$. Therefore, if such a scenario can work at all, it necessarily implies small $\tan \beta$. We leave a detailed investigation for future work.

\subsubsection{Cautionary note}

We stress that our conclusions here are specific to the MSSM, and attest to the high predictivity of the model. If the MSSM cannot survive in regions of metastability (where charge and colour-breaking minima exist but are not tunneled to over cosmological timescales), or be saved by higher-order corrections, more complicated supersymmetric models may still accommodate the excess, although the techniques described here may be useful in scrutinizing them. Another logical possibility of saving the MSSM would be production through the decay of heavier particles (say, stops, which could themselves be produced from gluino and squark decays). As mentioned in the beginning, the experimental data do not seem to support such a mechanism.

\section{Summary and outlook}

This work deals with the core phenomenology of the diphoton excess observed by the LHC experiments ATLAS and CMS around $750 \mathrm{GeV}$ diphoton invariant mass. We have considered both the case where the data are interpreted by a narrow and a broad resonance. We obtained model-independent constraints on the allowed couplings and branching fractions to various final states, including the interplay with other existing bounds. Our findings suggest that the anomaly cannot be accounted for by the presence of a single additional singlet or doublet spin-zero field and the Standard Model degrees of freedom; this includes all two-Higgs-doublet models. We also found that, at least in a leading-order analysis, the whole parameter space of the MSSM fails at explaining the excess if one requires the absence of charge and colour breaking minima. If we assume that the resonance is broad, we find that it is challenging to find a weakly coupled explanation. However, we provide an existence proof in the form of a model with vectorlike quarks with large electric charge. For the narrow resonance case, a similar model can be perturbative up to high scales also with smaller charges. We have also considered dilaton models where the full SM including the Higgs doublet is a part of the conformal sector. We find that these models cannot explain the size of the excess unless we add new fields below the $\mathrm{TeV}$ scale to give large extra contributions to the QED and QCD beta functions. As already mentioned, in all the scenarios studied by us we find that new particles below the $\mathrm{TeV}$ scale need to be present in addition to the resonance. They must have couplings to the scalar itself, to photons, maybe to gluons, and possibly also carry flavor information. Further study of their LHC 
phenomenology would be interesting to follow. Finally, models in which the new resonance has significant couplings to the light quarks motivate thinking about the linkage between flavor physics and the physics related to the resonance.

Note. Other early-response studies of the various possible implications of the excess, that appeared approximately simultaneously with ours, are refs. [57-73]. Also, after the submission, an earlier study of diphoton resonances [74] was pointed out to us.

\section{Acknowledgments}

GP is supported by the BSF, ISF, and ERC-2013-CoG grant (TOPCHARM \# 614794). SJ thanks GP and the Weizmann Institute for hospitality, including the period during which this paper was conceived. SJ acknowledges partial support from the U.K. STFC under Grant Agreement ST/L000504/1, and from the IPPP through an associateship. SJ acknowledges the NExT Institute.

Open Access. This article is distributed under the terms of the Creative Commons Attribution License (CC-BY 4.0), which permits any use, distribution and reproduction in any medium, provided the original author(s) and source are credited.

\section{References}

[1] ATLAS collaboration, Search for resonances decaying to photon pairs in $3.2 \mathrm{fb}^{-1}$ of $p p$ collisions at $\sqrt{s}=13 \mathrm{TeV}$ with the ATLAS detector, ATLAS-CONF-2015-081, CERN, Geneva Switzerland (2015).

[2] CMS collaboration, Search for new physics in high mass diphoton events in proton-proton collisions at $\sqrt{s}=13 \mathrm{TeV}$, CMS-PAS-EXO-15-004, CERN, Geneva Switzerland (2015).

[3] L.D. Landau, On the angular momentum of a system of two photons, Dokl. Akad. Nauk Ser. Fiz. 60 (1948) 207 [INSPIRE].

[4] C.-N. Yang, Selection rules for the dematerialization of a particle into two photons, Phys. Rev. 77 (1950) 242 [INSPIRE].

[5] J. Alwall et al., The automated computation of tree-level and next-to-leading order differential cross sections and their matching to parton shower simulations, JHEP 07 (2014) 079 [arXiv: 1405.0301] [INSPIRE].

[6] A. Alloul, N.D. Christensen, C. Degrande, C. Duhr and B. Fuks, FeynRules $2.0-a$ complete toolbox for tree-level phenomenology, Comput. Phys. Commun. 185 (2014) 2250 [arXiv: 1310.1921] [INSPIRE].

[7] S. Dawson, The effective W approximation, Nucl. Phys. B 249 (1985) 42 [InSPIRE].

[8] G.L. Kane, W.W. Repko and W.B. Rolnick, The effective $W^{ \pm}, Z^{0}$ approximation for high-energy collisions, Phys. Lett. B 148 (1984) 367 [INSPIRE].

[9] R.D. Ball et al., Parton distributions with LHC data, Nucl. Phys. B 867 (2013) 244 [arXiv: 1207.1303] [INSPIRE]. 
[10] C. Schmidt, J. Pumplin, D. Stump and C.P. Yuan, CT14QED parton distribution functions from isolated photon production in deep inelastic scattering, Phys. Rev. D 93 (2016) 114015 [arXiv: 1509.02905] [INSPIRE].

[11] L.A. Harland-Lang, V.A. Khoze and M.G. Ryskin, The production of a diphoton resonance via photon-photon fusion, JHEP 03 (2016) 182 [arXiv: 1601.07187] [INSPIRE].

[12] W. Altmannshofer, J. Galloway, S. Gori, A.L. Kagan, A. Martin and J. Zupan, $750 \mathrm{GeV}$ diphoton excess, Phys. Rev. D 93 (2016) 095015 [arXiv:1512.07616] [INSPIRE].

[13] S. Fichet, G. von Gersdorff and C. Royon, Scattering light by light at $750 \mathrm{GeV}$ at the LHC, Phys. Rev. D 93 (2016) 075031 [arXiv:1512.05751] [INSPIRE].

[14] CMS collaboration, Search for resonances decaying to dijet final states at $\sqrt{s}=8 \mathrm{TeV}$ with scouting data, CMS-PAS-EXO-14-005, CERN, Geneva Switzerland (2014).

[15] CMS collaboration, Search for diphoton resonances in the mass range from 150 to $850 \mathrm{GeV}$ in pp collisions at $\sqrt{s}=8 \mathrm{TeV}$, Phys. Lett. B $\mathbf{7 5 0}$ (2015) 494 [arXiv:1506.02301] [INSPIRE].

[16] ATLAS collaboration, Search for high-mass diphoton resonances in pp collisions at $\sqrt{s}=8 \mathrm{TeV}$ with the ATLAS detector, Phys. Rev. D 92 (2015) 032004 [arXiv:1504.05511] [INSPIRE].

[17] CMS collaboration, Search for high-mass diphoton resonances in pp collisions at $\sqrt{s}=8 \mathrm{TeV}$ with the CMS detector, CMS-PAS-EXO-12-045, CERN, Geneva Switzerland (2012).

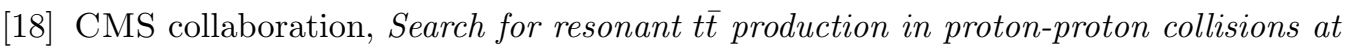
$\sqrt{s}=8$ TeV, Phys. Rev. D 93 (2016) 012001 [arXiv: 1506. 03062] [InSPIRE].

[19] ATLAS collaboration, Search for a high-mass Higgs boson decaying to a $W$ boson pair in $p p$ collisions at $\sqrt{s}=8 \mathrm{TeV}$ with the ATLAS detector, JHEP 01 (2016) 032 [arXiv: 1509.00389] [INSPIRE].

[20] CMS collaboration, Search for a Standard Model like Higgs boson in the $H \rightarrow Z Z \rightarrow \ell^{+} \ell^{-} q \bar{q}$ decay channel at $\sqrt{s}=8 \mathrm{TeV}$, CMS-PAS-HIG-14-007, CERN, Geneva Switzerland (2014).

[21] CMS collaboration, Search for di-Higgs resonances decaying to 4 bottom quarks, CMS-PAS-HIG-14-013, CERN, Geneva Switzerland (2014).

[22] ATLAS collaboration, Search for a new resonance decaying to a $W$ or $Z$ boson and a Higgs boson in the $\ell \ell / \ell \nu / \nu \nu+b \bar{b}$ final states with the ATLAS detector, Eur. Phys. J. C 75 (2015) 263 [arXiv: 1503.08089] [INSPIRE].

[23] ATLAS collaboration, Search for neutral Higgs bosons of the minimal supersymmetric Standard Model in pp collisions at $\sqrt{s}=8 \mathrm{TeV}$ with the ATLAS detector, JHEP 11 (2014) 056 [arXiv: 1409.6064] [inSPIRE].

[24] ATLAS collaboration, Search for new resonances in $W \gamma$ and $Z \gamma$ final states in pp collisions at $\sqrt{s}=8 \mathrm{TeV}$ with the ATLAS detector, Phys. Lett. B 738 (2014) 428 [arXiv:1407.8150] [INSPIRE].

[25] ATLAS collaboration, Search for high-mass dilepton resonances in pp collisions at $\sqrt{s}=8 \mathrm{TeV}$ with the ATLAS detector, Phys. Rev. D 90 (2014) 052005 [arXiv:1405.4123] [INSPIRE].

[26] CMS collaboration, Search for narrow resonances and quantum black holes in inclusive and b-tagged dijet mass spectra from pp collisions at $\sqrt{s}=7$ TeV, JHEP 01 (2013) 013 [arXiv:1210.2387] [INSPIRE]. 
[27] CMS collaboration, Search for heavy resonances decaying into bb and bg final states in pp collisions at $\sqrt{s}=8 \mathrm{TeV}$, CMS-PAS-EXO-12-023, CERN, Geneva Switzerland (2012).

[28] ATLAS collaboration, Search for new phenomena in dijet mass and angular distributions from pp collisions at $\sqrt{s}=13 \mathrm{TeV}$ with the ATLAS detector, Phys. Lett. B 754 (2016) 302 [arXiv: 1512.01530] [INSPIRE].

[29] CMS collaboration, Search for narrow resonances decaying to dijets in proton-proton collisions at $\sqrt{s}=13$ TeV, Phys. Rev. Lett. 116 (2016) 071801 [arXiv:1512.01224] [INSPIRE].

[30] W.D. Goldberger, B. Grinstein and W. Skiba, Distinguishing the Higgs boson from the dilaton at the Large Hadron Collider, Phys. Rev. Lett. 100 (2008) 111802 [arXiv:0708.1463] [INSPIRE].

[31] T. Robens and T. Stefaniak, Status of the Higgs singlet extension of the Standard Model after LHC run 1, Eur. Phys. J. C 75 (2015) 104 [arXiv:1501.02234] [INSPIRE].

[32] M. Spira, A. Djouadi, D. Graudenz and P.M. Zerwas, Higgs boson production at the LHC, Nucl. Phys. B 453 (1995) 17 [hep-ph/9504378] [inSPIRE].

[33] M. Son and A. Urbano, A new scalar resonance at $750 \mathrm{GeV}$ : towards a proof of concept in favor of strongly interacting theories, JHEP 05 (2016) 181 [arXiv:1512.08307] [INSPIRE].

[34] M.-L. Xiao and J.-H. Yu, Stabilizing electroweak vacuum in a vectorlike fermion model, Phys. Rev. D 90 (2014) 014007 [Addendum ibid. D 90 (2014) 019901] [arXiv:1404.0681] [INSPIRE].

[35] B. Gripaios, A. Pomarol, F. Riva and J. Serra, Beyond the minimal composite Higgs model, JHEP 04 (2009) 070 [arXiv: 0902 .1483] [INSPIRE].

[36] A. Efrati, E. Kuflik, S. Nussinov, Y. Soreq and T. Volansky, Constraining the Higgs-dilaton with LHC and dark matter searches, Phys. Rev. D 91 (2015) 055034 [arXiv:1410.2225] [INSPIRE].

[37] J.D. Wells, Lectures on Higgs boson physics in the Standard Model and beyond, in $39^{\text {th }}$ British Universities Summer School in Theoretical Elementary Particle Physics (BUSSTEPP 2009), Liverpool U.K. August 24-September 42009 [arXiv:0909.4541] [INSPIRE].

[38] B. Bellazzini, C. Csáki, J. Hubisz, J. Serra and J. Terning, A Higgslike dilaton, Eur. Phys. J. C 73 (2013) 2333 [arXiv:1209.3299] [INSPIRE].

[39] R.S. Gupta and J.D. Wells, Next generation Higgs bosons: theory, constraints and discovery prospects at the Large Hadron Collider, Phys. Rev. D 81 (2010) 055012 [arXiv:0912.0267] [INSPIRE].

[40] J.F. Gunion and H.E. Haber, The CP conserving two Higgs doublet model: the approach to the decoupling limit, Phys. Rev. D 67 (2003) 075019 [hep-ph/0207010] [INSPIRE].

[41] R.S. Gupta, M. Montull and F. Riva, SUSY faces its Higgs couplings, JHEP 04 (2013) 132 [arXiv:1212.5240] [INSPIRE].

[42] G.C. Branco, P.M. Ferreira, L. Lavoura, M.N. Rebelo, M. Sher and J.P. Silva, Theory and phenomenology of two-Higgs-doublet models, Phys. Rept. 516 (2012) 1 [arXiv:1106.0034] [INSPIRE]. 
[43] D. Ghosh, R.S. Gupta and G. Perez, Is the Higgs mechanism of fermion mass generation a fact? A Yukawa-less first-two-generation model, Phys. Lett. B 755 (2016) 504 [arXiv: 1508.01501] [INSPIRE].

[44] M. Drees and M.M. Nojiri, A new signal for scalar top bound state production, Phys. Rev. Lett. 72 (1994) 2324 [hep-ph/9310209] [INSPIRE].

[45] D. Kahawala and Y. Kats, Distinguishing spins at the LHC using bound state signals, JHEP 09 (2011) 099 [arXiv: 1103.3503] [INSPIRE].

[46] M.R. Kauth, J.H. Kuhn, P. Marquard and M. Steinhauser, Gluinonia: energy levels, production and decay, Nucl. Phys. B 831 (2010) 285 [arXiv:0910.2612] [INSPIRE].

[47] J.M. Frere, D.R.T. Jones and S. Raby, Fermion masses and induction of the weak scale by supergravity, Nucl. Phys. B 222 (1983) 11 [INSPIRE].

[48] J.P. Derendinger and C.A. Savoy, Quantum effects and $\mathrm{SU}(2) \times \mathrm{U}(1)$ breaking in supergravity gauge theories, Nucl. Phys. B 237 (1984) 307 [INSPIRE].

[49] J.A. Casas and S. Dimopoulos, Stability bounds on flavor violating trilinear soft terms in the MSSM, Phys. Lett. B 387 (1996) 107 [hep-ph/9606237] [INSPIRE].

[50] R. Rattazzi and U. Sarid, The unified minimal supersymmetric model with large Yukawa couplings, Phys. Rev. D 53 (1996) 1553 [hep-ph/9505428] [inSPIRE].

[51] J. Hisano and S. Sugiyama, Charge-breaking constraints on left-right mixing of stau's, Phys. Lett. B 696 (2011) 92 [Erratum ibid. B 719 (2013) 472] [arXiv: 1011. 0260] [INSPIRE].

[52] W. Altmannshofer, M. Carena, N.R. Shah and F. Yu, Indirect probes of the MSSM after the Higgs discovery, JHEP 01 (2013) 160 [arXiv:1211.1976] [INSPIRE].

[53] M. Carena, S. Gori, I. Low, N.R. Shah and C.E.M. Wagner, Vacuum stability and Higgs diphoton decays in the MSSM, JHEP 02 (2013) 114 [arXiv:1211.6136] [INSPIRE].

[54] W. Altmannshofer, C. Frugiuele and R. Harnik, Fermion hierarchy from sfermion anarchy, JHEP 12 (2014) 180 [arXiv: 1409.2522] [INSPIRE].

[55] A. Djouadi, The anatomy of electro-weak symmetry breaking. II. The Higgs bosons in the minimal supersymmetric model, Phys. Rept. 459 (2008) 1 [hep-ph/0503173] [InSPIRE].

[56] F. Borzumati, G.R. Farrar, N. Polonsky and S.D. Thomas, Soft Yukawa couplings in supersymmetric theories, Nucl. Phys. B 555 (1999) 53 [hep-ph/9902443] [INSPIRE].

[57] K. Harigaya and Y. Nomura, Composite models for the $750 \mathrm{GeV}$ diphoton excess, Phys. Lett. B 754 (2016) 151 [arXiv: 1512.04850] [INSPIRE].

[58] Y. Mambrini, G. Arcadi and A. Djouadi, The LHC diphoton resonance and dark matter, Phys. Lett. B 755 (2016) 426 [arXiv:1512.04913] [InSPIRE].

[59] M. Backovic, A. Mariotti and D. Redigolo, Di-photon excess illuminates dark matter, JHEP 03 (2016) 157 [arXiv: 1512.04917] [INSPIRE].

[60] A. Angelescu, A. Djouadi and G. Moreau, Scenarii for interpretations of the LHC diphoton excess: two Higgs doublets and vector-like quarks and leptons, Phys. Lett. B 756 (2016) 126 [arXiv: 1512.04921] [INSPIRE].

[61] Y. Nakai, R. Sato and K. Tobioka, Footprints of new strong dynamics via anomaly and the $750 \mathrm{GeV}$ diphoton, Phys. Rev. Lett. 116 (2016) 151802 [arXiv: 1512.04924] [INSPIRE]. 
[62] S. Knapen, T. Melia, M. Papucci and K. Zurek, Rays of light from the LHC, Phys. Rev. D 93 (2016) 075020 [arXiv: 1512.04928] [INSPIRE].

[63] D. Buttazzo, A. Greljo and D. Marzocca, Knocking on new physics' door with a scalar resonance, Eur. Phys. J. C 76 (2016) 116 [arXiv:1512.04929] [InSPIRE].

[64] A. Pilaftsis, Diphoton signatures from heavy axion decays at the CERN Large Hadron Collider, Phys. Rev. D 93 (2016) 015017 [arXiv:1512.04931] [INSPIRE].

[65] R. Franceschini et al., What is the $\gamma \gamma$ resonance at 750 GeV?, JHEP 03 (2016) 144 [arXiv: 1512.04933] [INSPIRE].

[66] S. Di Chiara, L. Marzola and M. Raidal, First interpretation of the $750 \mathrm{GeV}$ diphoton resonance at the LHC, Phys. Rev. D 93 (2016) 095018 [arXiv:1512.04939] [INSPIRE].

[67] T. Higaki, K.S. Jeong, N. Kitajima and F. Takahashi, The QCD axion from aligned axions and diphoton excess, Phys. Lett. B 755 (2016) 13 [arXiv:1512.05295] [InSPIRE].

[68] S.D. McDermott, P. Meade and H. Ramani, Singlet scalar resonances and the diphoton excess, Phys. Lett. B 755 (2016) 353 [arXiv:1512.05326] [InSPIRE].

[69] J. Ellis, S.A.R. Ellis, J. Quevillon, V. Sanz and T. You, On the interpretation of a possible $\sim 750 \mathrm{GeV}$ particle decaying into $\gamma \gamma$, JHEP 03 (2016) 176 [arXiv: 1512.05327] [INSPIRE].

[70] M. Low, A. Tesi and L.-T. Wang, A pseudoscalar decaying to photon pairs in the early LHC run 2 data, JHEP 03 (2016) 108 [arXiv:1512.05328] [INSPIRE].

[71] B. Bellazzini, R. Franceschini, F. Sala and J. Serra, Goldstones in diphotons, JHEP 04 (2016) 072 [arXiv: 1512.05330] [InSPIRE].

[72] C. Petersson and R. Torre, $750 \mathrm{GeV}$ diphoton excess from the Goldstino superpartner, Phys. Rev. Lett. 116 (2016) 151804 [arXiv: 1512.05333] [INSPIRE].

[73] E. Molinaro, F. Sannino and N. Vignaroli, Minimal composite dynamics versus axion origin of the diphoton excess, arXiv:1512.05334 [INSPIRE].

[74] J. Jaeckel, M. Jankowiak and M. Spannowsky, LHC probes the hidden sector, Phys. Dark Univ. 2 (2013) 111 [arXiv:1212.3620] [INSPIRE]. 